CITUSC/00-014 hep-th/0003100

\title{
Two-Time Physics in Field Theory
}

\author{
Itzhak Bars \\ CIT-USC Center for Theoretical Physics \\ and \\ Department of Physics and Astronomy \\ University of Southern California \\ Los Angeles, CA 90089-2535, USA
}

\begin{abstract}
A field theory formulation of two-time physics in $d+2$ dimensions is obtained from the covariant quantization of the constraint system associated with the $\operatorname{OSp}(n \mid 2)$ worldline gauge symmetries of two-time physics. Interactions among fields can then be included consistently with the underlying gauge symmetries. Through this process a relation between Dirac's work in 1936 on conformal symmetry in field theory and the more recent worldline formulation of two-time physics is established while providing a worldline gauge symmetry basis for the field equations in $d+2$ dimensions. It is shown that the field theory formalism goes well beyond Dirac's goal of linearizing conformal symmetry. In accord with recent results in the worldline approach of two-time physics, the $d+2$ field theory can be brought down to diverse $d$ dimensional field theories by solving the subset of field equations that correspond to the "kinematic" constraints. This process embeds the one "time" in $d$-dimensions in different ways inside the $d+2$ dimensional spacetime. Thus, the two-time $d+2$ field theory appears as a more fundamental theory from which many one-time $d$ dimensional field theories are derived. It is suggested that the hidden symmetries and relations among computed quantities in certain $d$-dimensional interacting field theories can be taken as the evidence for the presence of a higher unifying structure in a $d+2$ dimensional spacetime. These phenomena have similarities with ideas such as dualities, AdS-CFT correspondence and holography.
\end{abstract}

\footnotetext{
${ }^{1}$ This research was partially supported by the US. Department of Energy under grant number DE-FG0384ER40168.
} 


\section{Introduction}

In 1936 Dirac invented a field theory approach for rewriting conformal field theory in four dimensions in a manifestly $\mathrm{SO}(4,2)$ covariant form in six dimensions [1]. Dirac's fields $\Phi(X)$ depend on 6 coordinates $X^{M}$ which have two timelike dimensions, just like the dynamical coordinates $X^{M}(\tau, \cdots)$ used in the formalism of two-time physics on the worldline or worldvolume [2]-[10]. In the notation of [2]-[10] to label $X^{M}$, with $M=+^{\prime},-^{\prime}, 0,1,2,3$, Dirac's choice of coordinates are as follows: Minkowski space coordinates $x^{\mu}$ are the homogeneous coordinates $x^{\mu}=X^{\mu} / X^{+^{\prime}}$, with $\mu=0,1,2,3$, while the extra coordinate $X^{-^{\prime}}$ is eliminated through the $\mathrm{SO}(4,2)$ invariant constraint $X \cdot X=-2 X^{+^{\prime}} X^{{{ }^{\prime}}^{\prime}}+X_{\mu} X^{\mu}=0$. The extra coordinates $X^{0^{\prime}}, X^{1^{\prime}}$ given by $X^{ \pm^{\prime}}=\left(X^{0^{\prime}} \pm X^{1^{\prime}}\right) / \sqrt{2}$ describe one extra timelike and one extra spacelike dimensions. Dirac showed that the free field equations for scalar, fermion and vector fields in 4 dimensions $\phi\left(x^{\mu}\right)$ can be rewritten $\mathrm{SO}(4,2)$ covariantly in terms of fields $\Phi\left(X^{M}\right)$ that depend on the 6 coordinates, provided these fields also satisfy additional $\mathrm{SO}(4,2)$ covariant subsidiary conditions. Several authors pursued Dirac's idea and extended it to interacting conformal field theories, including conformally invariant Yang-Mills theories [1] [12], but then Dirac's idea was forgotten for a long time. Recently this approach has been applied to conformal gravity and its interactions with conformal matter [13].

Dirac's goal was to realize conformal symmetry linearly in $4+2$ dimensional field theory, and this remained the primary motivation for the work in the literature that followed his paper. The goals and results of two-time physics lie in more general directions, although conformal symmetry is included as a special outcome in a particular gauge. In two-time physics there is an underlying new gauge principle that is responsible for recasting the $d+2$ dimensional theory as many possible $d$-dimensional theories. The purpose of the present paper is twofold. First, to establish the relationship between the gauge principles in two time physics on the worldline and Dirac's approach in field theory; second, to demonstrate directly in field theory that diverse one-time field theories emerge in $d$ dimensions from the same field equations in $d+2$ dimensions. It will be seen that the path of derivation of $d$ dimensional field theories is in precise correspondence with making gauge choices in the worldline theory, the important step being the embedding of the time coordinate in $d$ dimensions in various ways inside the $d+2$ dimensions. In this way one can see that the $d+2$ dimensional two-time theory plays a unifying role in a new sense, including interactions.

Two-time physics in $d+2$ dimensions was developed independently in the worldline (and worldvolume) formulation [2]-[10], unaware of the field theory formalism invented by Dirac which had been long forgotten 2. It was perhaps lucky that ignorance of Dirac's approach permitted the free exploration and development of new insights in the worldline formulation

\footnotetext{
${ }^{2}$ I thank Vasilev for bringing to my attention his recent work, and informing me of Dirac's work and the line of research that followed the same trend of thought in relation to conformal symmetry.
} 
that were not necessarily connected with conformal symmetry. Historically, the motivation for two-time physics came from duality, and signals for two-timelike dimensions in M-theory and its extended superalgebra including D-branes [14]- 24]. In particular certain dynamical attempts [20] 22] to try to understand these phenomena directly paved the way to the formalism in [2]. Two-time physics introduced a new gauge principle - $\operatorname{Sp}(2, R)$ in phase space, and its generalizations - that insures unitarity, causality and absence of ghosts. This takes care of problems that naively would have arisen in a spacetime with two-timelike dimensions. Morally speaking, this gauge symmetry is related to duality in a generalized sense. The new phenomenon in two-time physics is that this gauge symmetry can be used to obtain various one-time dynamical systems in $d$ dimensions from the same two-time action in $d+2$ dimensions, through gauge fixing, thus uncovering a new layer of unification through higher dimensions. In this paper we will show that the same insights can be expressed in the language of field theory.

First we will show that the $\operatorname{Sp}(2, R)$ gauge symmetry (or $\operatorname{OSp}(n \mid 2)$ for spinning particles) provides a fundamental gauge symmetry basis for Dirac's field equations in $d+2$ dimensions. In effect, the field equations amount to imposing the non-Abelian $\operatorname{OSp}(n \mid 2)$ constraints in an $\mathrm{SO}(d, 2)$ covariant quantization of the worldline-two-time physics theory, while the fields represent the gauge invariant states. After reaching a two-time field theory formalism for scalars, spinors, vectors and higher spin fields, field interactions consistent with the underlying worldline gauge invariance is included. In particular, interactions that are local in $d+2$ spacetime, such as Yang-Mills or general reparametrizations, must satisfy certain "kinematic" field equations beyond the dynamical field equations, that are in complete agreement with recent results obtained through background field methods in two-time physics on the worldline [10]. The interacting field theory constructed in this way is in agreement with the latest developments in the Dirac approach included in [13].

Second, it is shown that, depending on the path of coming down from $d+2$ dimensions to some chosen subset of $d$ dimensions, by solving the "kinematic" subset of the field equations, the physical meaning of the one-time field theory, as interpreted by an observer in the remaining $d$ dimensions, can be quite different. In particular the natural $\mathrm{SO}(d, 2)$ Lorentz symmetry of the original field equations (in the case of flat $d+2$ dimensional spacetime) can be interpreted in different ways depending on the choice of the remaining $d$ coordinates. The resulting onetime field theory has conformal symmetry if one follows Dirac's path from $d+2$ to $d$, but with various embeddings of $d$ dimensions in $d+2$ dimensions one arrives at various one-time field theories. In the flat case, all resulting $d$ dimensional field theories have new hidden $\operatorname{SO}(d, 2)$ symmetries which are not necessarily conformal symmetries. Thus the two-time field theory approach unifies classes of one-time physical systems in $d$ dimensions that previously would have been thought of as being described by $d$-dimensional field theories unrelated to each other.

Solving the "kinematic" subset of field equations amounts to a gauge choice in the worldline formalism of two-time physics, and therefore the physical interpretation of the remaining field 
theory agrees with similar recent results in the worldline approach. The main essential new point achieved through field theory is the inclusion of interactions in this new type of unification.

These results hold at the level of classical field theory, which could be thought of as the first quantization of the worldline theory. To extend them to second quantized field theory (and analyze issues such as anomalies, etc.) certain open problems in the field theoretic formulation of two-time physics need to be understood. These may involve non-commutative geometry, and they are briefly discussed in the last section. Analogies and connections with other concepts in the literature, such as duality, AdS-CFT and holography are also pointed out in the last section.

\section{Local and global symmetry}

The two-time worldline description of particle dynamics, in the absence of background fields (i.e. "free" case[), is given by the $S p(2, R)$ gauge theory described by the action [2]

$$
\begin{aligned}
S_{0} & =\frac{1}{2} \int d \tau D_{\tau} X_{i}^{M} X_{j}^{N} \varepsilon^{i j} \eta_{M N} \equiv \int d \tau\left(\partial_{\tau} X_{1}^{M} X_{2}^{N}-\frac{1}{2} A^{i j} X_{i}^{M} X_{j}^{N}\right) \eta_{M N} \\
& =\int d \tau\left(\partial_{\tau} X^{M} P^{N}-\frac{1}{2} A^{11} X^{M} X^{N}-\frac{1}{2} A^{22} P^{M} P^{N}-A^{12} X^{M} P^{N}\right) \eta_{M N} .
\end{aligned}
$$

Here $X_{i}^{M}(\tau)$ is an $S p(2, R)$ doublet, consisting of the ordinary coordinate and its conjugate momentum $\left(X_{1}^{M} \equiv X^{M}\right.$ and $\left.X_{2}^{M} \equiv P^{M}=\partial S_{0} / \partial X_{1 M}\right)$. The indices $i, j=1,2$ denote the doublet $S p(2, R)$, they are raised and lowered by the antisymmetric Levi-Civita symbol $\varepsilon_{i j}$. The gauge covariant derivative $D_{\tau} X_{i}^{M}$ that appears in (1) is defined as

$$
D_{\tau} X_{i}^{M}=\partial_{\tau} X_{i}^{M}-\varepsilon_{i k} A^{k l} X_{l}^{M} .
$$

The local $S p(2, R)$ acts as $\delta X_{i}^{M}=\varepsilon_{i k} \omega^{k l} X_{l}^{M}$ and $\delta A^{i j}=\omega^{i k} \varepsilon_{k l} A^{l j}+\omega^{j k} \varepsilon_{k l} A^{i l}+\partial_{\tau} \omega^{i j}$, where $\omega^{i j}(\tau)$ is a symmetric matrix containing the three $\operatorname{Sp}(2, R)$ gauge parameters and $A^{i j}$ is the gauge field on the worldline. The second form of the action (2) is obtained after an integration by parts so that only $X_{1}^{M}$ appears with derivatives. This allows the identification of $X, P$ by the canonical procedure, as indicated in the third form of the action.

The gauge fields $A^{11}, A^{12}=A^{21}$, and $A^{22}$ act as Lagrange multipliers for the following three first class constraints that form the $\operatorname{Sp}(2, R)$ algebra

$$
X_{i} \cdot X_{j}=0 \rightarrow X^{2}=P^{2}=X \cdot P=0,
$$

\footnotetext{
${ }^{3}$ Although interactions are not explicitly present in the "free" action in $d+2$ dimensions, the solution of the constraints generates a class of dynamics for the remaining degrees of freedom in $d$ dimensions after a gauge is fixed. When background fields are present all possible particle dynamics in $d$ dimensions (rather than only a class) can be described from the point of view of two-time physics in $d+2$ dimensions, as shown in [10]. We also mention that another generalization is space-time supersymmetry, including a generalized local kappa supersymmetry [5] [6] [9]. This enriches both the local symmetries as well as the global symmetries. The formalism has also been generalized to strings and branes with limited success so far [7] (although full success is expected).
} 
as implied by the local $S p(2, R)$ invariance. It is precisely the solution of these constraints that require that the global metric $\eta_{M N}$ has a signature with two-time like dimensions. Thus, $\eta_{M N}$ stands for the flat metric on a $(d, 2)$ dimensional space-time, which is the only signature consistent with the equations of motion for the $\operatorname{Sp}(2, R)$ gauge field $A^{k l}$, leading to non-trivial dynamics that can be consistently quantized. Hence the global two-time $S O(d, 2)$ is implied by the local $\operatorname{Sp}(2, R)$ symmetry.

The explicit global $S O(d, 2)$ invariance has the Lorentz generators

$$
L^{M N}=X^{M} P^{N}-X^{N} P^{M}=\varepsilon^{i j} X_{i}^{M} X_{j}^{N}
$$

that are manifestly $\operatorname{Sp}(2, R)$ gauge invariant. As mentioned above, different gauge choices lead to different particle dynamics in $d$ dimensions (relativistic massless and massive particles, nonrelativistic massive particle, H-atom, harmonic oscillator, particle in $\operatorname{AdS}_{d-k} \times \mathrm{S}^{k}$ background etc.) all of which have $S O(d, 2)$ invariant actions that are directly obtained from (1) by gauge fixing. Since the action (11) and the generators $L^{M N}$ (5) are gauge invariant, the global symmetry $\mathrm{SO}(d, 2)$ is not lost by gauge fixing. This explains why one should expect a hidden (previously unnoticed) global symmetry $\mathrm{SO}(d, 2)$ for each of the systems that result by gauge fixing [3].

To describe spinning particles, worldline fermions $\psi_{a}^{M}(\tau)$, with $a=1,2, \cdots, n$ are introduced. Together with $X^{M}, P^{M}$, they form the fundamental representation $\left(\psi_{a}^{M}, X^{M}, P^{M}\right)$ of $\operatorname{OSp}(n \mid 2)$. Gauging this supergroup [4] instead of $\operatorname{Sp}(2, R)$ produces a Lagrangian that has $n$ local supercharges plus $n$ local conformal supercharges on the worldline, in addition to local $\operatorname{Sp}(2, R)$ and local $\mathrm{SO}(n)$. The full set of first class constraints that correspond to the generators of these gauge symmetries are, at the classical level,

$$
X \cdot X=P \cdot P=X \cdot P=X \cdot \psi_{a}=P \cdot \psi_{a}=\psi_{[a} \cdot \psi_{b]}=0 .
$$

To have non-trivial classical solutions of these constraints (with angular momentum) at least two timelike dimensions are required. The $\operatorname{OSp}(n \mid 2)$ gauge symmetry can remove the ghosts of no more than two timelike dimensions. Therefore, as in the spinless case, the signature is fixed and the global symmetry of the theory is $\operatorname{SO}(d, 2)$. It is applied to the label $M$ in $\left(\psi_{a}^{M}, X^{M}, P^{M}\right)$. The global $\operatorname{SO}(d, 2)$ generators $J^{M N}$ that commute with all the $\operatorname{OSp}(n \mid 2)$ gauge generators (6) are

$$
J^{M N}=L^{M N}+S^{M N}, \quad S^{M N}=\frac{1}{2 i}\left(\psi_{a}^{M} \psi_{a}^{N}-\psi_{a}^{N} \psi_{a}^{M}\right) .
$$

In this paper we will be interested in the covariant quantization of the theory in a manifestly $\mathrm{SO}(d, 2)$ covariant formalism. This will be used in the next section to construct the $d+2$ dimensional field theory. The commutation rules are

$$
\left[X^{M}, P^{N}\right]=i \eta^{M N}, \quad\left\{\psi_{a}^{M}, \psi_{b}^{N}\right\}=\eta^{M N} \delta_{a b},
$$


while all other commutators among the basic degrees of freedom are zero. The $S p(2, R)$ or $\operatorname{OSp}(n \mid 2)$ gauge constraints applied on the Hilbert space are just enough to remove all negativenorm states ("ghosts") introduced by the two timelike dimensions [2] [4], resulting in a unitary quantum theory. We will treat spinless particles as a special case of $\operatorname{OSp}(n \mid 2)$ with $n=0$, so we will state the covariant quantization procedure directly for $\operatorname{OSp}(n \mid 2)$.

Since the constraints form a non-Abelian algebra one must choose a commuting subset of operators to label the Hilbert space. In particular the local $\operatorname{OSp}(n \mid 2)$ labels and the global $\mathrm{SO}(d, 2)$ labels correspond to simultaneously diagonalizable operators that include the Casimir operators of both groups

$$
\mid O S p(n \mid 2) \text { labels; SO }(d, 2) \text { labels }>
$$

The $\operatorname{OSp}(n \mid 2)$ quadratic Casimir operator that commutes with all the generators in (6) is (before they are set to zero)

$$
\begin{aligned}
C_{2}(O S p(n \mid 2))= & \frac{1}{8}\left(X^{2} P^{2}+P^{2} X^{2}\right)-\frac{1}{16}(X \cdot P+P \cdot X)^{2} \\
& +\frac{1}{4 i}\left(X \cdot \psi_{a} P \cdot \psi_{a}-P \cdot \psi_{a} X \cdot \psi_{a}\right) \\
& +\frac{1}{32}\left(\psi_{[a} \cdot \psi_{b]}\right)\left(\psi_{[a} \cdot \psi_{b]}\right) .
\end{aligned}
$$

On the other hand, the global $\mathrm{SO}(d, 2)$ quadratic Casimir operator is given by (orders of operators respected)

$$
\begin{aligned}
C_{2}(S O(d, 2)) & =\frac{1}{2} J^{M N} J_{M N}=\frac{1}{2} L^{M N} L_{M N}+\frac{1}{2} S^{M N} S_{M N}+L^{M N} S_{M N} \\
\frac{1}{2} L^{M N} L_{M N} & =\frac{1}{2}\left(X^{2} P^{2}+P^{2} X^{2}\right)-\frac{1}{4}(X \cdot P+P \cdot X)^{2}+1-\frac{d^{2}}{4} \\
\frac{1}{2} S^{M N} S_{M N} & =\frac{1}{8}\left(\psi_{[a} \cdot \psi_{b]}\right)\left(\psi_{[a} \cdot \psi_{b]}\right)+\frac{1}{8} n(d+2)(d+n) \\
L^{M N} S_{M N} & =-i\left(X \cdot \psi_{a} P \cdot \psi_{a}-P \cdot \psi_{a} X \cdot \psi_{a}\right)-\frac{1}{2} n(d+2) .
\end{aligned}
$$

The extra constants arise from the re-ordering of quantum operators. In the last two lines we have used $\psi_{a} \cdot \psi_{a}=n(d+2) / 2$ that follows from the quantum relation. We see that the Casimir operator of $\mathrm{SO}(d, 2)$ is related to the Casimir operator of $\operatorname{OSp}(n \mid 2)$

$$
C_{2}(S O(d, 2))=4 C_{2}(O S p(n \mid 2))+\frac{1}{8}(d+2)(n-2)(d+n-2) .
$$

Similarly, higher Casimir operators of $S O(d, 2)$ are also related to Casimir operators of $\operatorname{OSp}(n \mid 2)$ except for ordering constants.

One must demand that the physical states be singlets under the gauge symmetry $\operatorname{OSp}(n \mid 2)$. This requires vanishing Casimir operators of the gauge group, in particular $C_{2}(O S p(n \mid 2))=0$. 
This leads to definite and unique eigenvalues for the $\mathrm{SO}(d, 2)$ Casimir operators for physical states. Thus, on physical states the quadratic Casimir operator must have the eigenvalue

$$
C_{2}(S O(d, 2))=\frac{1}{8}(n-2)(d+2)(d+n-2) .
$$

Similarly, the higher Casimir eigenvalues for $\mathrm{SO}(d, 2)$ are also fixed. Therefore, for given $d, n$ one must take a specific $\mathrm{SO}(d, 2)$ representation to guarantee an $\mathrm{OSp}(n \mid 2)$ gauge singlet. For example, for spinless particles $(n=0)$ the quadratic Casimir is fixed to $C_{2}=1-d^{2} / 4$ (in the absence of background fields).

When the quantization is performed in a fixed gauge the same eigenvalue of the Casimir operators must emerge for the dynamics of the remaining dynamical system in $d$ dimensions for a fixed $n$. Indeed after careful ordering of non-linear products of operators this is verified explicitly (see [2] [4] for examples of non-covariant quantization in several fixed gauges). The covariant quantization explains why seemingly unrelated dynamics in $d$ dimensions (such as massless relativistic particle, H-atom, harmonic oscillator in one less dimension, particle in $\mathrm{AdS}_{d-k} \times \mathrm{S}^{k}$ for all $k$, etc.) all must realize the same unitary representation of $\mathrm{SO}(d, 2)$, as they indeed do.

\section{Fields, "kinematics" and "dynamics"}

If the system is quantized in a fixed gauge, one time and one space dimensions are eliminated, making the absence of ghosts and the one-time nature of the system quite evident [2] [3]. The quantum theory is then expressed in terms of a wave equation in $d$ dimensions for each one of the fixed gauges (e.g. for $n=0$ spinless particles: Klein-Gordon, non-relativistic Schrödinger, H-atom wave equation, Klein-Gordon in $\mathrm{AdS}_{d-k} \times \mathrm{S}^{k}$ background, etc.). Each one of these wave equations is derivable from an effective field theory action in $d$ dimensions. These field theory actions look different but yet they all represents the quantum theory of the same $d+2$ system. Since the original theory had an $\mathrm{SO}(d, 2)$ global symmetry, the derived field theories, although they look different, must all have $\mathrm{SO}(d, 2)$ global symmetry and they must all be related. A well

known case of the symmetry is the conformal $\mathrm{SO}(d, 2)$ symmetry of the massless Klein-Gordon theory. The symmetry must be present for all the others, and indeed it is the case, provided one takes care of anomalies produced by quantum ordering of operators. For example, the particle on $\operatorname{AdS}_{d-k} \times \mathrm{S}^{k}$ background would not be $\mathrm{SO}(d, 2)$ symmetric (for every $k$ ) at the field theory level unless a quantized mass term produced by quantum ordering is included in the action [3].

Similar comments apply for spin $1 / 2$ wave equations, such as the Dirac equation, etc. produced by the various gauge fixings of the $\operatorname{OSp}(1 \mid 2)$ gauge theory, or for spin 1 wave equations, such as Maxwell equation etc. produced by the various gauge fixings of the $\operatorname{OSp}(2 \mid 2)$ theory.

An interesting question is: Is there a master field theory in $d+2$ dimensions from which all of these $d$ dimensional field theories are derived by a procedure akin to the gauge fixing in 
the underlying $\operatorname{OSp}(n \mid 2)$ theory? Furthermore, if field interactions are added to each of the $d$ dimensional theories, which of these interactions would still represent the unified master field theory in $d+2$ dimensions, thereby making the different $d$ dimensional theories all equivalent to each other under some kind of duality transformation?

These questions are answered by quantizing the worldline theory in a manifestly $\mathrm{SO}(d, 2)$ covariant formalism. The wave equation is then in $d+2$ dimensions, and it is supplemented by additional field equations that we call "kinematic" as opposed to "dynamic" field equations. The "kinematic" equations impose a subset of the underlying $\operatorname{OSp}(n \mid 2)$ constraints. The "dynamic" field equations correspond to another subset of constraints, but are derived from a field theory action in $d+2$ dimensions. Field interactions are included in this dynamic action. When the kinematic equations are solved, the field theory is reduced from $d+2$ dimensions to $d$ dimensions, but there is a choice of which $d$ dimensions among $d+2$ survive in the remaining field equations. This choice is equivalent to the gauge fixing that could be done in the worldline formulation of the theory. Indeed the remaining $d$ dimensional field theory that comes from the $d+2$ field theory correctly produces the wave equations derived from the gauge fixed worldline theory, including any anomalies. But now the consistent interactions are also fixed for the $d$ dimensional version of the theory, since they all come directly from the field interactions in $d+2$ dimensions.

The formulation of the $d+2$ field equations, both kinematic and dynamic, proceeds as follows. A physical state $\mid \Phi>$ of the worldline theory is labelled by both $\operatorname{OSp}(n \mid 2)$ and $\operatorname{SO}(d, 2)$ (if no background fields) as in (9). The $\operatorname{OSp}(n \mid 2)$ labels must correspond to a singlet for a gauge invariant physical state. The $\operatorname{OSp}(n \mid 2)$ labels include a set of commuting generators in addition to the $\operatorname{OSp}(n \mid 2)$ Casimir eigenvalues that are zero. On a physical state that is $\operatorname{OSp}(n \mid 2)$ singlet the $\mathrm{SO}(n)$ generators given by $\frac{1}{2 i} \psi_{[a} \cdot \psi_{b]}$ must all vanish (since the physical state must be an $\mathrm{SO}(n)$ singlet). There is an exception for $n=2$ : the $\mathrm{SO}(2)$ generator $\frac{1}{2 i} \psi_{[1} \cdot \psi_{2]}=q$ need not vanish since every representation of $\mathrm{SO}(2)$ is a singlet (although not neutral if $q \neq 0$ ). In addition, among the set of commuting operators in $\operatorname{OSp}(n \mid 2)$ that would vanish on a singlet, one is tempted to choose the generators $P^{2}$ and $P \cdot \psi_{a}$ since these would produce Klein-Gordon and Dirac equations. If these operators vanish we would be forced into a free field theory. However, before we impose this last condition, let us re-examine the expression of the Casimir operator (10) to find out if we can make a weaker choice. As we will see, this is indeed the case, and the weaker choice will allow us to include interactions in field theory.

The $\operatorname{OSp}(n \mid 2)$ Casimir (10) may be rewritten by pulling $P^{2}$ and $P \cdot \psi_{a}$ to the right side

$$
\begin{aligned}
C_{2}(O S p(n \mid 2))= & \frac{1}{4}\left(i X \cdot P+\frac{d+2}{2}+|q| \delta_{n, 2}\right)\left(i X \cdot P+\frac{d-2}{2}+n-|q| \delta_{n, 2}\right) \\
& +\frac{1}{4} X^{2} P^{2}-\frac{i}{2} X \cdot \psi_{a} P \cdot \psi_{a}+\frac{1}{32}\left(\psi_{[a} \cdot \psi_{b]}\right)\left(\psi_{[a} \cdot \psi_{b]}\right)
\end{aligned}
$$

To define a physical state, with a vanishing $C_{2}(O S p(n \mid 2))=0$, it is sufficient to simultaneously diagonalize the commuting operators $i X \cdot P, X^{2} P^{2}, X \cdot \psi_{a} P \cdot \psi_{a}$ all of which commute also with 
the $\mathrm{SO}(n)$ generators $\frac{1}{2 i} \psi_{[a} \cdot \psi_{b]}$. Thus, a physical state is defined by

$$
\begin{gathered}
X^{2} P^{2}\left|\Phi>=0, \quad\left(i X \cdot P+\frac{d-2}{2}+n-|q| \delta_{n, 2}\right)\right| \Phi>=0 \\
X \cdot \psi_{a} P \cdot \psi_{a}\left|\Phi>=0, \quad\left(\frac{1}{2 i} \psi_{[a} \cdot \psi_{b]}-q \delta_{n, 2} \varepsilon_{a b}\right)\right| \Phi>=0 .
\end{gathered}
$$

Demanding an $\operatorname{OSp}(n \mid 2)$ singlet also imposes the $\mathrm{SO}(d, 2)$ Casimir eigenvalue given in (18). Some additional operators, even if they do not commute with the above, may have definite eigenvalues on physical states $|\Phi\rangle$, since we are interested in the states that give only the zero eigenvalues of the operators above rather than all of their eigenvalues. It may then be quantum mechanically compatible if certain additional operators take on specific values as well on the physical states (for example, even though the $\mathrm{SO}(n)$ generators do not commute with each other they can all vanish simultaneously on a $\mathrm{SO}(n)$ singlet).

In addition to the physical ket states $\mid \Phi>$ we also consider the spin and position space bra states $<X, \operatorname{spin} \mid$. The probability amplitude $<X, \operatorname{spin} \mid \Phi>\equiv \Phi_{\text {spin }}(X)$ defines the physical fields or wavefunctions that will enter in the $d+2$ dimensional field theory. The spin labels will be explained below. On the state $<X$, spin $\mid$ the position operators $X^{M}$ are diagonal. An important property of this state is defined by demanding the $X^{2}$ operator to vanish $<$ $X, \operatorname{spin} \mid X^{2}=0$ as a constraint imposed on the position Hilbert space. From

$$
0=<X, \operatorname{spin}\left|X^{2}\right| \Phi>\equiv X^{2} \Phi_{\text {spin }}(X)
$$

we learn that $\Phi_{\text {spin }}(X)$ vanishes everywhere, except on the $d+2$ dimensional lightcone where $X^{2}=0$. Therefore, to examine the non-trivial fields we must take $X^{2}=0$. On position space the momentum operators act as derivatives $<X$, spin $\mid P^{M}=-i \partial_{M}<X$, spin $\mid$. The quantization procedure we have just adopted (i.e. imposing $X^{2}$ on bra states) implies that when there are derivatives applied on the fields, such as $\partial_{M} \Phi_{\text {spin }}(X)$, the derivative must be performed first before imposing the constraint

$$
X^{2}=0
$$

This describes one of the "kinematic" equations that will be needed. Another kinematic constraint is the second equation in (20). On the fields it takes the form

$$
\left(X \cdot \partial+\frac{d-2}{2}+n-|q| \delta_{n, 2}\right) \Phi_{\text {spin }}(X)=0 .
$$

where $|q|$ will be related to the spin in the case of $n=2$. Basically this requires fields of specific scales depending on their spin. The required scale is in $d+2$ dimensions, not in $d$ dimensions. A third kinematic equation is the second equation in (21), but we will solve that one completely and the fields $\Phi_{\text {spin }}(X)$ will be defined after the explicit solution of that equation.

There remains the "dynamic" equations, the first equations in (20,21), which yield KleinGordon or Dirac type equations for the fields $\Phi_{\text {spin }}(X)$. In the next few sections we study the 
dynamic equations for each spinning field $\Phi_{\text {spin }}(X)$, include field interactions, and build an action from which they can be derived. The combination of the interacting field theory action and the kinematic equations $(23,24)$ define the $d+2$ dimensional field theory at the classical level.

\section{Scalar field $(n=0)$}

For $n=0\left(\operatorname{drop} \psi_{a}^{M}\right)$ the worldline theory based on $\operatorname{OSp}(0 \mid 2)=S p(2, R)$ describes a spinless particle. The dynamic (20) and kinematic equations (23,24) take the form

$$
X^{2} \partial^{M} \partial_{M} \Phi(X)=0, \quad X^{M} \partial_{M} \Phi(X)=-\frac{d-2}{2} \Phi(X), \quad X^{2} \Phi(X)=0,
$$

Consistent interactions have the form

$$
\partial^{M} \partial_{M} \Phi=\lambda \Phi^{(d+2) /(d-2)}+\cdots
$$

where $\cdots$ stands for interactions with other fields that we will discuss below. All interactions are constrained by demanding consistency with the $\operatorname{Sp}(2, R)$ kinematic constraints in (25), which are imposed by applying $X \cdot \partial$ or $X^{2}$ on both sides, and using (25). Without the interactions this equation is consistent with choosing to diagonalize $P^{2} \sim 0$ on the physical state, which was possible in the first place, but by going through the steps above we see that $\partial^{M} \partial_{M} \Phi(X)$ need not vanish while remaining consistent with the physical state conditions. In general, if written in radial coordinates, the Laplacian operator $\partial^{M} \partial_{M}$ in $d+2$ dimensions has terms proportional to $1 / X^{2}$, which will tend to blow up as $X^{2} \rightarrow 0$

$$
\partial^{M} \partial_{M}=\frac{1}{X^{2}}\left((X \cdot \partial)^{2}+d X \cdot \partial-\frac{1}{2} L^{M N} L_{M N}\right)
$$

but the numerator is zero after using the second equation in (25) and the physical value of the $\mathrm{SO}(d, 2)$ Casimir (13) for $n=0$. Therefore the operator $\partial^{M} \partial_{M} \sim 0 / 0$ is finite on a physical state as given in (26). In this way we have seen that the underlying $S p(2, R)$ gauge symmetry permits only certain interactions. If $d+2=6$ (i.e. $d=4$ ) the right hand side of (26) contains $g \Phi^{3}$. The field equation can be derived from the variation of the Lagrangian

$$
L_{d+2}^{\Phi}=-\frac{1}{2} \Phi \partial^{M} \partial_{M} \Phi-\lambda \frac{(d-2)}{2 d} \Phi^{2 d /(d-2)},
$$

and it must be supplemented by the subsidiary kinematic conditions in (25).

Evidently one can write a richer $d+2$ field theory involving several scalar fields that have interactions with each other so long as those interactions are consistent with the subsidiary kinematic conditions. This means that the power $2 d /(d-2)$ should be saturated, but this can be done by the product of several scalar fields. If $d=4$ the interaction if $\Phi^{4}$, but other powers are not permitted. 
The equations in (25) are a slight generalization of Dirac's equations [1] that he obtained by a different set of arguments (instead of the first eq. in (25) he had $\partial^{M} \partial_{M} \Phi(X)=0$ ). In our case these equations follow directly from the $\operatorname{Sp}(2, R)$ gauge symmetry conditions of the worldline theory, and thus provide a gauge theory basis for Dirac's approach.

We will next solve the subsidiary kinematic field equations and show that the remaining dynamics is described by a field theory in $d$ dimensions. However, we will see that there are many ways of choosing coordinates in coming from $d+2$ dimensions down to $d$ dimensions while solving the subsidiary conditions. The choice of coordinates is parallel to fixing a $\operatorname{Sp}(2, R)$ gauge in the worldline theory. Various one-time field theories in $d$ dimensions emerge when "time" is identified in different ways within the $d+2$ dimensional space. One of those cases corresponds to conformal field theory, with $\mathrm{SO}(d, 2)$ interpreted as the conformal group, as Dirac suggested. However, all other choices of coordinates lead to other $d$ dimensional field theories with $\operatorname{SO}(d, 2)$ symmetry, but with $\mathrm{SO}(d, 2)$ taking on different meanings as less familiar hidden symmetries. Thus, the content of these field equations goes well beyond the linearization of conformal symmetry envisaged by Dirac and the literature that followed his path [1] [11] [12] [13]. In fact, the equations above unify a class of different looking $d$-dimensional one-time field theories into the same $d+2$ dimensional two-time field theory, including interactions, as shown below.

\subsection{Massless scalar field in d dimensions}

In the worldline formulation the gauge fixing $X^{+}(\tau)=1$ and $P^{+}(\tau)=0$, and solution of constraints $X^{2}=0$ and $X \cdot P=0$ left behind the Minkowski coordinates and momenta $x^{\mu}(\tau)$, $p^{\mu}(\tau)$ as the independent degrees of freedom

$$
\begin{aligned}
X^{+^{\prime}}(\tau) & =1, \quad X^{-^{\prime}}(\tau)=x^{2} / 2, \quad X^{\mu}=x^{\mu}(\tau), \\
P^{+^{\prime}}(\tau) & =0, \quad P^{{-^{\prime}}^{\prime}}(\tau)=x \cdot p, \quad P^{\mu}=p^{\mu}(\tau)
\end{aligned}
$$

constrained only by $p^{2}=0$. The dynamics of the remaining coordinates describe the massless relativistic particle [2]. The quantization of the remaining system produced the Klein-Gordon equation which in turn can be derived from the Klein-Gordon action that has the $\mathrm{SO}(d, 2)$ conformal symmetry identified with the Lorentz symmetry $L^{M N}=X^{M} P^{N}-X^{N} P^{M}$ in $d+2$ dimensions [2]. Field interactions may then be added, but there is no specific instructions for which interactions are permitted, unless one tries to maintain the $\mathrm{SO}(d, 2)$ Lorentz symmetry.

Now, let us do the analog of this gauge fixing directly in the $d+2$ dimensional field theory of the previous section. Following Dirac, we use the change of variables

$$
X^{+^{\prime}}=\kappa, \quad X^{-^{\prime}}=\kappa \lambda, \quad X^{\mu}=\kappa x^{\mu}
$$

where the one-time is embedded in Minkowski space $x^{\mu}$ while the dependence on the other time will be determined by solving the kinematic field equations. Using the chain rule, $\partial_{M}=$ 
$\frac{\partial \kappa}{\partial X^{M}} \frac{\partial}{\partial \kappa}+\frac{\partial \lambda}{\partial X^{M}} \frac{\partial}{\partial \lambda}+\frac{\partial x^{\mu}}{\partial X^{M}} \frac{\partial}{\partial x^{\mu}}$ we find

$$
\begin{aligned}
\frac{\partial}{\partial X^{+^{\prime}}} & =\frac{\partial}{\partial \kappa}-\frac{\lambda}{\kappa} \frac{\partial}{\partial \lambda}-\frac{x^{\mu}}{\kappa} \frac{\partial}{\partial x^{\mu}} \\
\frac{\partial}{\partial X^{-^{\prime}}} & =\frac{1}{\kappa} \frac{\partial}{\partial \lambda}, \quad \frac{\partial}{\partial X^{\mu}}=\frac{1}{\kappa} \frac{\partial}{\partial x^{\mu}}
\end{aligned}
$$

Note that $P^{+^{\prime}}$ (which was set to zero as a gauge choice in the worldline approach) is represented by the derivative operator

$$
P^{+^{\prime}}=-P_{-^{\prime}}=i \frac{\partial}{\partial X^{-^{\prime}}}=\frac{1}{\kappa} \frac{\partial}{\partial \lambda}
$$

At this stage no gauge choices have been made;= only a change to more convenient coordinates has been performed, but note the parallel with the gauge in (29,30). Next, we can write the differential operators in the new coordinates

$$
\begin{aligned}
X^{M} \partial_{M}= & \kappa \frac{\partial}{\partial \kappa} \\
\partial^{M} \partial_{M}= & \frac{1}{\kappa^{2}}\left(\frac{\partial}{\partial x^{\mu}}+x_{\mu} \frac{\partial}{\partial \lambda}\right)^{2}-\frac{1}{\kappa^{2}}\left(2 \kappa \frac{\partial}{\partial \kappa}+d-2\right) \frac{\partial}{\partial \lambda} \\
& +\frac{1}{\kappa^{2}}\left(2 \lambda-x^{2}\right)\left(\frac{\partial}{\partial \lambda}\right)^{2}
\end{aligned}
$$

These differential operators are to be applied on a physical field which is parametrized as $\Phi\left(\kappa, \lambda, x^{\mu}\right)$ before imposing the kinematic constraints $X^{2}=0$. To impose this constraint one must set $\lambda=x^{2} / 2$ after differentiation $\frac{\partial}{\partial \lambda}$. Then we see that the third term in $\partial^{M} \partial_{M}$ drops out on sufficiently non-singular wavefunctions. Using the kinematic constraint in (25) together with (35) the kappa dependence is fully determined as an overall factor $\kappa^{-(d-2) / 2}$

$$
\Phi(X)=\kappa^{-(d-2) / 2} f(x, \lambda) .
$$

This solution allows us to drop also the second term in $\partial^{M} \partial_{M}$. Next, note that derivatives with respect to $x^{\mu}$ appear only in the combination $\frac{\partial}{\partial x^{\mu}}+x_{\mu} \frac{\partial}{\partial \lambda}$. Then, setting $\lambda=x^{2} / 2$ after differentiation using the derivative operator $\frac{\partial}{\partial x^{\mu}}+x_{\mu} \frac{\partial}{\partial \lambda}$ gives the same result as setting $\lambda=x^{2} / 2$ before differentiation and differentiating only with $\frac{\partial}{\partial x^{\mu}}$

$$
\left[\left(\frac{\partial}{\partial x^{\mu}}+x_{\mu} \frac{\partial}{\partial \lambda}\right) f(x, \lambda)\right]_{\lambda=x^{2} / 2}=\frac{\partial}{\partial x^{\mu}} f\left(x, \frac{x^{2}}{2}\right) .
$$

Therefore we can set $\left.f(x, \lambda)\right|_{\lambda=x^{2} / 2}=\phi(x)$ before differentiation provided we also drop the term $\frac{\partial}{\partial \lambda}$ in the derivative operator $\frac{\partial}{\partial x^{\mu}}+x_{\mu} \frac{\partial}{\partial \lambda}$. We see that all $\frac{\partial}{\partial \lambda}$ terms have dropped out from the Laplace operator $\partial^{M} \partial_{M}$ in (36). The disappearance of $\frac{\partial}{\partial \lambda}$ everywhere is parallel to setting $P^{+^{\prime}}=0$ as a $\operatorname{Sp}(2, R)$ gauge choice as in (30,34). Using these remarks we see that the physical state conditions (25,26) are by now fully solved in this gauge by the following general form

$$
\Phi(X)=\kappa^{-(d-2) / 2} \phi(x), \quad \frac{\partial^{2} \phi(x)}{\partial x^{\mu} \partial x_{\mu}}=\lambda \phi^{(d+2) /(d-2)},
$$


where $\phi(x)$ is an interacting massless Klein-Gordon field in $d$-dimensional Minkowski spacetime (in this interaction we assumed a single real field, but it could be more general). The effective action that generates this equation of motion is

$$
L_{d}^{\phi}=-\frac{1}{2} \phi \partial^{\mu} \partial_{\mu} \phi-\frac{\lambda(d-2)}{8 d} \phi^{2 d /(d-2)} .
$$

This is in full agreement with the effective field theory that was obtained by quantizing the worldline formalism in the fixed gauge $X^{+^{\prime}}(\tau)=1, P^{+^{\prime}}(\tau)=0$, as given in [2].

Note that the $d+2$ Lagrangian (28) reduces directly to the $d$ Lagrangian (40) when the solution of the subsidiary conditions (39) and the form of the Laplacian (36) are used

$$
L_{d+2}^{\Phi}(X) \rightarrow \kappa^{-d} L_{d}^{\phi}(x)
$$

$\kappa$ disappears after integration over $\kappa$ in the action.

Thus, solving just the kinematic equations $X^{2}=0$ and $X \cdot \partial \Phi=-\frac{1}{2}(d-2) \Phi$ with a particular choice of the remaining $d$ coordinates, and replacing the solution into the $\mathrm{SO}(d, 2)$ invariant action is sufficient to obtain the dynamics and the interpretation of the theory in $d$ dimensions.

It is well known that the interacting massless Klein-Gordon theory (40), including the interaction, is invariant under conformal transformations, although the symmetry is somewhat "hidden". In the two time formalism given above the conformal symmetry is inherited from the manifestly $\mathrm{SO}(d, 2)$ invariant equations $(25,26)$ as shown in different ways in [1] and [2]. This allows us to interpret conformal symmetry in $d$ dimensions as the Lorentz symmetry in $d+2$ dimensions acting on the space $X^{M}$.

Thus conformal symmetry in (39) can be taken as evidence for an underlying higher space with one more timelike and one more spacelike dimensions. In this higher spacetime all $d+2$ dimensions are at an equal footing - it is only because of the asymmetric choice of coordinates $\kappa, \lambda, x^{\mu}$ that (i) the remaining one "time" $x^{0}$ was defined and (ii) the manifest symmetry was broken artificially in the process of solving the "kinematic" equations (gauge constraints) to rewrite the $d+2$ field equations as a field theory in $d$ dimensions.

The more unifying aspect of the higher space, and the interpretation of the hidden symmetry as being simply the higher Lorentz symmetry, will make a stronger impression on the reader after noting that a similar observation is repeated in several seemingly unrelated field theoretic models that are actually derivable from the same set of field theoretic equations in the higher dimensions. Each of the derived field theories in $d$ dimensions has the $\operatorname{SO}(d, 2)$ symmetry realized in the same irreducible unitary representation, but its interpretation is not conformal symmetry. Nevertheless, it is the same Lorentz symmetry of the higher $d+2$ spacetime. 


\subsection{Scalar field in $\mathbf{A d S}_{D} \times S^{k}$ background}

To show that the meaning of $\mathrm{SO}(d, 2)$ goes beyond the conformal symmetry interpretation, let us now demonstrate that the same $\mathrm{SO}(d, 2)$ invariant equations (25,26) have a different physical interpretation when the coordinates, in particular "time", are chosen in a different way. Let the $d+2=D+k+2$ coordinates be labelled as $X^{M}=\left(X^{+^{\prime}}, X^{-^{\prime}}, X^{\mu}, X^{i}\right)$ with $X^{\mu}$ representing $(D-1)$ spacetime dimensions with one time, and $X^{i}$ representing $k+1$ spacelike dimensions, so $D+k=d$. Consider the following change of variables (this is related to the $\operatorname{Sp}(2, R)$ gauge choice for a particle moving in the $\operatorname{AdS}_{D} \times S^{k}$ background in the worldline formalism as given in [3])

$$
\begin{aligned}
X^{+^{\prime}} & =\rho u, \quad X^{{\iota^{\prime}}^{\prime}}=\rho \sigma, \quad X^{i}=\rho \frac{\mathbf{u}^{i}}{u} a, \quad X^{\mu}=\frac{1}{a} \rho u x^{\mu} . \\
\rho & =\frac{\sqrt{X_{i}^{2}}}{a}, \quad \sigma=\frac{a X^{-^{\prime}}}{\sqrt{X_{i}^{2}}}, \quad \mathbf{u}^{i}=\frac{a X^{+^{\prime}} X^{i}}{X_{i}^{2}}, \quad x^{\mu}=\frac{a X^{\mu}}{X^{+^{\prime}}}
\end{aligned}
$$

The $\mathbf{u}^{i}$ are Euclidean vectors in $k+1$ dimensions, $u$ is the magnitude of the Euclidean vector $u=|\mathbf{u}|, a$ is a constant with dimension of length, and $x^{\mu}$ are Minkowski vectors in $(D-1)$ dimensions. The $X^{2}=0$ condition gives

$$
\sigma=\frac{a^{4}+x^{2} u^{2}}{2 u a^{2}}
$$

The $\mathrm{SO}(d, 2)$ covariant line element in $d+2$ dimensions $d X \cdot d X$ gives the $\operatorname{AdS}_{D} \times S^{k}$ line element in $D+k=d$ dimensions up to a conformal factor (after using (44)),

$$
\begin{aligned}
d X \cdot d X & =\rho^{2}\left(\frac{(d \mathbf{u})^{2}}{u^{2}}+\frac{u^{2}}{a^{2}}\left(d x_{\mu}\right)^{2}\right) \\
& =\rho^{2}\left(\left(d \Omega_{k}\right)^{2}+\frac{d u^{2}}{u^{2}}+\frac{u^{2}}{a^{2}}\left(d x_{\mu}\right)^{2}\right),
\end{aligned}
$$

where $\left(d \Omega_{k}\right)^{2}$ is the metric on $S^{k}$. This shows the relationship of the parametrization to the $\operatorname{AdS}_{D} \times S^{k}$ background, with $D+k=d$. We will consider all possible values of $k=$ $0,1, \cdots,(d-2)$, so that we will exhibit a relation among the field theories for fixed $d$, written on the backgrounds $\mathrm{AdS}_{d}, \mathrm{AdS}_{d-1} \times S^{1}, \cdots, \mathrm{AdS}_{2} \times S^{d-2}$.

Let us rewrite (25,26) in these coordinates. The chain rule $\partial_{M}=\left(\partial_{M} \rho\right) \frac{\partial}{\partial \rho}+\left(\partial_{M} \sigma\right) \frac{\partial}{\partial \sigma}+$ $\left(\partial_{M} \mathbf{u}^{i}\right) \frac{\partial}{\partial \mathbf{u}^{i}}+\left(\partial_{M} x^{\mu}\right) \frac{\partial}{\partial x^{\mu}}$ gives

$$
\begin{aligned}
\frac{\partial}{\partial X^{+^{\prime}}} & =\frac{1}{\rho u}\left(\mathbf{u}^{i} \frac{\partial}{\partial \mathbf{u}^{i}}-x^{\mu} \frac{\partial}{\partial x^{\mu}}\right), \quad \frac{\partial}{\partial X^{-^{\prime}}}=\frac{1}{\rho} \frac{\partial}{\partial \sigma}, \quad \frac{\partial}{\partial X^{\mu}}=\frac{a}{\rho u} \frac{\partial}{\partial x^{\mu}} \\
\frac{\partial}{\partial X^{i}} & =\frac{\mathbf{u}^{i}}{a u}\left(\frac{\partial}{\partial \rho}-\frac{\sigma}{\rho} \frac{\partial}{\partial \sigma}-2 \frac{\mathbf{u}^{j}}{\rho} \frac{\partial}{\partial \mathbf{u}^{j}}\right)+\frac{u}{a \rho} \frac{\partial}{\partial \mathbf{u}^{i}}
\end{aligned}
$$


Using these, the relevant differential operators $X^{M} \partial_{M}, \partial_{M} \partial^{M}$ (before using (44)) may be written in the form

$$
\begin{aligned}
X^{M} \partial_{M} & =\rho \frac{\partial}{\partial \rho} \\
\partial_{M} \partial^{M} & =\frac{a^{2}}{\rho^{2} u^{2}}\left(D_{\mu}\right)^{2}+\left[\frac{\mathbf{u}^{i}}{a u} \frac{\partial}{\partial \rho}+\frac{u}{a \rho}\left(\mathbf{D}_{i}-2 \frac{\mathbf{u}^{i}}{u} \frac{\mathbf{u}^{j}}{u} \mathbf{D}_{j}\right)\right]^{2}+\cdots
\end{aligned}
$$

where the derivative operators $D_{\mu}, \mathbf{D}_{i}$ are given by

$$
D_{\mu}=\frac{\partial}{\partial x^{\mu}}+\frac{u x_{\mu}}{a^{2}} \frac{\partial}{\partial \sigma}, \quad \mathbf{D}_{i}=\frac{\partial}{\partial \mathbf{u}^{i}}+\frac{\mathbf{u}^{i}}{u}\left(\frac{x^{2}}{2 a^{2}}-\frac{a^{2}}{2 u^{2}}\right) \frac{\partial}{\partial \sigma},
$$

and the terms $\cdots$ are all proportional to $\left(2 u a^{2} \sigma-a^{4}-x^{2} u^{2}\right)$ which vanishes according to (44).

The general solution of the second equation in (25) now takes the form

$$
\Phi(X)=\left.\rho^{-(d-2) / 2} F(\sigma, \mathbf{u}, x)\right|_{\sigma=\left(a^{4}+x^{2} u^{2}\right) / 2 a^{2} u}
$$

We note again that it is possible to replace the differential operators $D_{\mu}, \mathbf{D}_{i}$ that are applied before the substitution $\sigma=\left(a^{4}+x^{2} u^{2}\right) / 2 u a^{2}$ with the simple differentiation $\frac{\partial}{\partial x^{\mu}}, \frac{\partial}{\partial \mathbf{u}^{i}}$ if the substitution is done before differentiation

$$
\begin{aligned}
{\left[D_{\mu} F(\sigma, \mathbf{u}, x)\right]_{\sigma=\left(a^{4}+x^{2} u^{2}\right) / 2 u a^{2}} } & =\frac{\partial}{\partial x^{\mu}} F\left(\frac{a^{4}+x^{2} u^{2}}{2 u a^{2}}, \mathbf{u}, x\right) \\
{\left[\mathbf{D}_{i} F(\sigma, \mathbf{u}, x)\right]_{\sigma=\left(a^{4}+x^{2} u^{2}\right) / 2 u a^{2}} } & =\frac{\partial}{\partial \mathbf{u}^{i}} F\left(\frac{a^{4}+x^{2} u^{2}}{2 u a^{2}}, \mathbf{u}, x\right)
\end{aligned}
$$

Therefore, we may define the field $\phi(x, \mathbf{u})$ that depends only on the $\operatorname{AdS}_{d-k} \times S^{k}$ variables $x^{m}=\left(x^{\mu}, \mathbf{u}^{i}\right)$

$$
\left.\phi(x, \mathbf{u}) \equiv F(\sigma, \mathbf{u}, x)\right|_{\sigma=\left(a^{4}+x^{2} u^{2}\right) / 2 a^{2} u}
$$

Combined with the vanishing of the $\cdots$ terms in $(50)$ the net effect is to drop the derivatives $\partial / \partial \sigma$ wherever they appear. This is equivalent to the choice of the $\operatorname{Sp}(2, R)$ gauge $P^{+^{\prime}}=$ $\frac{\partial}{i \partial X^{-^{\prime}}}=\frac{1}{i \rho} \frac{\partial}{\partial \sigma}=0$ which was performed in the worldline formalism [3]. With these remarks, we then find that the full set of equations (25, 26, 49, 50) are solved provided $\phi(x, \mathbf{u})$ satisfies the scalar equation in the $\mathrm{AdS}_{d-k} \times S^{k}$ background with a quantized mass term

$$
\begin{aligned}
\Phi(X) & =\rho^{-(d-2) / 2} \phi(x, \mathbf{u}) \\
0 & =\frac{1}{\sqrt{-G}} \partial_{m}\left(\sqrt{-G} G^{m n} \partial_{n} \phi\right)+M^{2} \phi+\lambda \phi^{(d+2) /(d-2)} \\
M^{2} & \equiv \frac{1}{4 a^{2}}(d-2)(d-2 k),
\end{aligned}
$$

where the metric $G_{m n}$ is given by the $\operatorname{AdS}_{d-k} \times S^{k}$ line element, with labels $x^{m}=\left(x^{\mu}, \mathbf{u}^{i}\right)$

$$
d s^{2}=\frac{(d \mathbf{u})^{2}}{u^{2}}+\frac{u^{2}}{a^{2}}\left(d x_{\mu}\right)^{2} \equiv G_{m n} d x^{m} d x^{n}
$$


Note that the mass term vanishes if $d=2$ or if $d=2 k$. Thus for $\operatorname{AdS}_{2} \times S^{d-2}$ and $\operatorname{AdS}_{d / 2} \times S^{d / 2}$ there is no mass term. These equations follow from the Lagrangian in $d$ total dimensions

$$
\begin{aligned}
L_{d}^{\phi}= & -\frac{1}{2} \phi \partial_{m}\left(\sqrt{-G} G^{m n} \partial_{n} \phi\right) \\
& -\sqrt{-G}\left[\frac{(d-2)(d-2 k)}{8 a^{2}} \phi^{2}+\frac{\lambda(d-2)}{2 d} \phi^{2 d /(d-2)}\right]
\end{aligned}
$$

This Lagrangian also follows directly from the $d+2$ dimensional Lagrangian by inserting the solution of the kinematic constraints given in (56)

$$
L_{d+2}^{\Phi}=\rho^{-d} L_{d}^{\phi}
$$

The $\rho$ dependence disappears in the action after an integration of the Lagrangian in $d+2$ dimensions.

The same result was derived in [3] by choosing a $\operatorname{Sp}(2, R)$ gauge in the worldline formalism and then doing non-covariant quantization. There, it was essential to figure out the correct ordering of the quantum operators, which in turn gave rise to the quantized mass given above. Thus, the quantized mass term is a quantum anomaly. If the anomaly is missed, the $\operatorname{AdS}_{d-k} \times \mathrm{S}^{k}$ theory would no longer be equivalent to the $d+2$ dimensional theory or any of the other $d$ dimensional versions.

The evident symmetry of this action is only $\mathrm{SO}(d-k-1,2) \times S O(k+1)$ which corresponds to the Killing symmetries of the $\operatorname{AdS}_{d-k} \times S^{k}$ metric $G_{m n}$. However, there is more hidden symmetry in this action that was not noticed before the advent of two-time physics [3]. In the present field theory setting this follows simply from the property that the original set of equations (25, 26) are invariant under the larger $\mathrm{SO}(d, 2)$. This contains the Killing symmetries as a subgroup, but the total symmetry is larger. Therefore we should expect that there are hidden symmetries in the effective action that correspond to the additional generators in the coset

$$
\frac{S O(d, 2)}{S O(d-k-1,2) \times S O(k+1)} .
$$

That is, the effective action given above should have the full $\mathrm{SO}(d, 2)=S O(D+k, 2)$ symmetry for every $k$. Indeed it was shown in [3], that this action has the full symmetry $\operatorname{SO}(d, 2)$. The quantized mass term is essential for this symmetry to be valid. Hence, the larger symmetry requires a quantized mass. The generators of the full symmetry, and the transformation of $\phi(x, \mathbf{u})$ under them are explicitly given for every $k$ in [3]. The presence of the symmetry is again evidence for the underlying larger space that contains one more spacelike and more timelike dimensions.

Through this example, with various $k$, we have demonstrated that the content of the fully covariant equations $(25,26)$ is much more than the conformal massless particle that was originally aimed for by Dirac [1]. The field theoretic results reported here fully agree with the 
worldline formalism at the quantum level performed also at fixed gauges [3. Furthermore, in the field theory formalism field interactions consistent with the $\mathrm{SO}(d, 2)$ symmetry are also introduced directly in $d+2$ theory.

It is interesting to consider the AdS-CFT correspondence [25]- [27] in this setting. Going to the boundary of AdS corresponds to $u \rightarrow \infty$. In this limit the original form of the theory in $d+2$ dimensions can be analyzed easily by examining the parametrization given in (42). We may also define $\rho=\kappa / u$ to more easily extract the information when we take the limit with finite $\kappa$. In this limit the coordinates and momenta have the form

$$
X^{+^{\prime}} \rightarrow \kappa, \quad X^{{-^{\prime}}^{\prime}} \rightarrow \frac{\kappa x^{2}}{2 a^{2}}, \quad X^{i} \rightarrow 0, \quad X^{\mu} \rightarrow \frac{\kappa}{a} x^{\mu}
$$

We see that the $d+2$ space shrinks in the $k+1$ dimensions $X^{i}$, and remains finite in the $d-k-1$ dimensions $X^{\mu}$. Then the two-time Lagrangian (28) gets reduced $L_{d+2}^{\Phi} \rightarrow L_{d-k+1}^{\Phi}$ in the number of dimensions. By comparison to the parametrization of the previous section, and recalling eqs. 39-41), we learn that the full field theory given by $L_{d+2}^{\Phi}$ now shrinks to a conformal field theory in $d-k-1$ dimensions that defines the boundary of the AdS space

$$
L_{d+2}^{\Phi} \rightarrow L_{d-k+1}^{\Phi}=\kappa^{(d-k-1) / 2} L_{d-k-1}^{\phi} .
$$

This is precisely the AdS-CFT correspondence applied to this theory. Having the two-time theory in the form $L_{d+2}^{\Phi}$ as the common link for various parametrizations, permitted the analysis to proceed in a straightforward manner in proving the AdS-CFT correspondence in the present case.

\subsection{Non-relativistic Schrödinger field}

The two cases, massless Klein-Gordon and particle in $\mathrm{AdS} \times \mathrm{S}$ discussed in the two previous sections are relatively easy from the point of view of operator ordering in the first quantized theory. In this section we would like to discuss a harder case in which it is not a priori evident how to order quantum operators.

In the worldline theory the gauge fixing $P^{+^{\prime}}(\tau)=m$, and $P^{0}(\tau)=0$ at the classical level produces the non-relativistic massive particle with mass $m$ [3]. In this gauge the remaining degrees of freedom are designated by the canonical pairs $(t(\tau), H(\tau))$ and $\left(\mathbf{r}^{i}(\tau), \mathbf{p}^{i}(\tau)\right)$ which

are constrained by $H=\mathbf{p}^{2} / 2 m$. These are related to the $X^{M}, P^{M}$ which satisfy $X^{2}=0$ and $X \cdot P=0$ as follows

$$
P^{+^{\prime}}=m, \quad P^{-^{\prime}}=H(\tau), \quad P^{0}=0, \quad P^{i}=\mathbf{p}^{i}(\tau)
$$

where $m$ is a $\tau$ independent constant, and

$$
\begin{aligned}
X^{+^{\prime}} & =t(\tau), \quad X^{{{ }^{\prime}}^{\prime}}=\frac{1}{m}(\mathbf{r} \cdot \mathbf{p}-t H), \quad X^{i}=\mathbf{r}^{i}(\tau), \\
X^{0} & = \pm \sqrt{\mathbf{r}^{2}-\frac{2 t}{m}(\mathbf{r} \cdot \mathbf{p}-t H)}
\end{aligned}
$$


The $H=\mathbf{p}^{2} / 2 m$ condition follows from the remaining dynamical constraint $P^{2}=0$. Evidently the field theory version of this dynamical constraint is the Schrödinger equation

$$
i \frac{\partial \phi(t, \mathbf{r})}{\partial t}=-\frac{1}{2 m} \nabla^{2} \phi(t, \mathbf{r})+\cdots
$$

that follows from the free Lagrangian in $d$ dimensions

$$
L_{d}^{\phi}=i \phi^{*} \frac{\partial \phi}{\partial t}-\frac{1}{2 m} \nabla \phi^{*} \nabla \phi+\cdots
$$

The $\operatorname{dots} \cdots$ represent interactions that could be added.

The non-relativistic particle action $S=\int d t \frac{m}{2}\left(\partial_{t} \mathbf{r}\right)^{2}$ has a surprising $\operatorname{SO}(d, 2)$ symmetry (non-conformal) given by the gauge fixed form of the global $\mathrm{SO}(d, 2)$ Lorentz generators $L^{M N}=$ $X^{M} P^{N}-X^{N} P^{M}$ as explained in [3]. Evidently the field theory that is derived from the $d+2$ field theory must also inherit this symmetry. Operator ordering of the quantity $X^{0}(68)$ is non-trivial, and therefore constructing the $\mathrm{SO}(d, 2)$ symmetry generators $L^{M N}$ at the quantum level in this fixed gauge is not easy. These $L^{M N}$ would be the Noether charges for the symmetry $\mathrm{SO}(d, 2)$ of the Schrödinger theory. The corresponding problem in the previous two cases were solved satisfactorily by fixing the correct anomalies [3], but the non-linear form of (68) has discouraged the analysis so far. How does this problem show up in the field theory version, and how is it resolved, in particular when there are field interactions? Without a guiding symmetry such as $\mathrm{SO}(d, 2)$ there would not be restrictions on the interactions.

Let us now try to imitate directly in the $d+2$ dimensional field theory the gauge fixing $P^{+^{\prime}}(\tau)=m$, and $P^{0}(\tau)=0$ of the worldline theory. Before applying any of the kinematic constraints, the relevant $\mathrm{SO}(d, 2)$ differential operators can be rewritten in the form

$$
X^{M} \partial_{M}=X^{+^{\prime}} D_{+^{\prime}}+X^{-^{\prime}} D_{-^{\prime}}+X^{i} D_{i}-X^{M} X_{M} \frac{1}{X^{0}} \partial_{0}
$$

and

$$
\partial^{M} \partial_{M}=-2 D_{+^{\prime}} D_{-^{\prime}}+\left(D_{i}\right)^{2}-\frac{2}{X^{0}} \partial_{0}\left(X^{M} \partial_{M}+\frac{d-2}{2}\right)-X^{M} X_{M}\left(\frac{1}{X^{0}} \partial_{0}\right)^{2}
$$

where

$$
D_{+^{\prime}}=\partial_{+^{\prime}}-\frac{X^{{-^{\prime}}^{\prime}}}{X^{0}} \partial_{0}, \quad D_{-^{\prime}}=\partial_{-^{\prime}}-\frac{X^{+^{\prime}}}{X^{0}} \partial_{0}, \quad D_{i}=\partial_{i}+\frac{X_{i}}{X^{0}} \partial_{0}
$$

Imposing $X^{M} X_{M}=0$ is equivalent to setting

$$
X_{0}= \pm \sqrt{X^{i} X_{i}-2 X^{+^{\prime}} X^{-^{\prime}}}
$$

but, before doing so, we must apply all the derivatives $\partial_{0}$ on the wavefunction $\Phi\left(X^{0}, X^{+^{\prime}}, X^{{{ }^{\prime}}^{\prime}}, X^{i}\right)$. However, from $(71,72$ ) we see that when the kinematic constraints are applied all terms containing the explicit $\partial_{0}$ drop out, except those appearing in the definition of $D_{+^{\prime}}, D_{-^{\prime}}, D_{i}$. Furthermore, for these special combinations, applying first the derivative and then imposing (74)

\footnotetext{
${ }^{4}$ Bo Zhang first constructed the following formulas. I thank him for showing me his work.
} 
gives the same result as first imposing (74) and replacing $D_{+^{\prime}}, D_{-^{\prime}}, D_{i}$ with ordinary derivatives $\partial_{+^{\prime}}, \partial_{-^{\prime}}, \partial_{i}$

$$
\left[D_{i, \pm^{\prime}} \Phi\left(X^{0}, X^{+^{\prime}}, X^{-^{\prime}}, X^{i}\right)\right]_{X^{0}= \pm \sqrt{X^{i} X_{i}-2 X^{+^{\prime} X^{-^{\prime}}}}}=\left.\partial_{i, \pm^{\prime}} \Phi\right|_{X^{0}}
$$

where we have defined the notation

$$
\left.\Phi\right|_{X^{0}} \equiv \Phi\left( \pm \sqrt{X^{i} X_{i}-2 X^{+^{\prime}} X^{-^{\prime}}}, X^{+^{\prime}}, X^{{-^{\prime}}^{\prime}}, X^{i}\right)
$$

In this way $\partial_{0}$ completely disappears and $X^{0}$ is expressed in terms of the other coordinates. This is the field theory version of the gauge condition $P^{0}(\tau)=0$ used in the worldline approach.

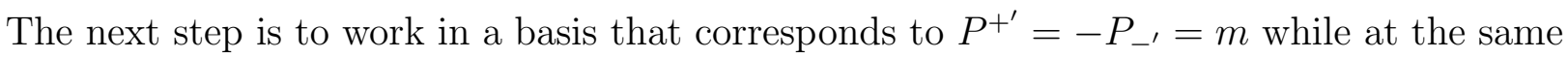
time solve the remaining kinematic constraint that now takes the form

$$
\left.\left(X^{+^{\prime}} \partial_{+^{\prime}}+X^{-^{\prime}} \partial_{-^{\prime}}+X^{i} \partial_{i}+\frac{d-2}{2}\right) \Phi\right|_{X^{0}}=0
$$

This is done by first going to Fourier space in the $X^{-^{\prime}}$ coordinate and then imposing the kinematic constraint. The result is

$$
\left.\Phi\right|_{X^{0}}=\int d m e^{-i m X^{-^{\prime}}} m^{(d-4) / 2} \phi\left(m X^{+^{\prime}}, m X^{i}\right)
$$

where the function $\phi\left(t, \mathbf{r}^{i}\right)$ is arbitrary. Finally we apply the dynamical operator on this form and find the Schrödinger operator

$$
\begin{aligned}
\left(\partial^{M} \partial_{M} \Phi\right) \| & =\left(-2 \partial_{+^{\prime}} \partial_{-^{\prime}}+\partial_{i} \partial^{i}\right)\left(\left.\Phi\right|_{X^{0}}\right) \\
& =\int d m e^{-i m X^{-^{\prime}}} m^{(d-4) / 2}\left[\left(2 i m \frac{\partial}{\partial t}+\nabla_{\mathbf{r}}^{2}\right) \phi(t, \mathbf{r})\right]_{t=m X^{+^{\prime}}, \mathbf{r}^{i}=m X^{i}}
\end{aligned}
$$

On the left side the notation $\left(\partial^{M} \partial_{M} \Phi\right) \|$ implies that both kinematic constraints have been implemented.

Now we see that the free field equation in $d+2$ dimensions $\left(\partial^{M} \partial_{M} \Phi\right) \|=0$ corresponds to

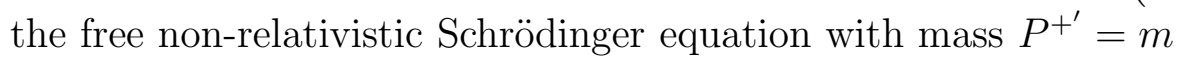

$$
i \frac{\partial}{\partial t} \phi(t, \mathbf{r})=-\frac{1}{2 m} \nabla_{\mathbf{r}}^{2} \phi(t, \mathbf{r})
$$

in agreement with the first quantization of the worldline theory given in (69). By rewriting it in the form $\left(\partial^{M} \partial_{M} \Phi\right) \|=0$ the hidden $\operatorname{SO}(d, 2)$ symmetry of the Schrödinger equation is exposed. The interactions consistent with the $\mathrm{SO}(d, 2)$ symmetry follow from the original equations in $d+2$ dimensions, but unfortunately they do not seem to have a simple or recognizable form in this case, so we will not discuss it any longer in this paper. 


\subsection{Generalizations}

As argued above, a class of one-time physics dynamics is unified by the field theoretic two-time formalism. The class is much larger than the cases discussed above since, as we know from the worldline approach, it includes other one-time dynamics such as the H-atom, harmonic oscillator, particle in various potentials, etc. [3]. It would be interesting to explore the interacting field theory for some of these cases. The interaction term then provides a field theoretic approach to the interaction of these systems in a setting which has never been explored before. In some generalized sense this is analogous to duality in M-theory.

The effective one-time field theories thus obtained, the ones in the previous sections, as well as any others derived similarly in other embeddings of $d$ dimensions inside the $d+2$ spacetime, are all representatives of the same two-time field theory which provides for some remarkable relations among them. Such relations were not apparent before the insight provided by two-time physics [2]-[10]. In principle, in related $d$ dimensional field theories one should be able to compute $\operatorname{Sp}(2, R)$ gauge invariant quantities and obtain the same result. The $\mathrm{SO}(d, 2)$ symmetry properties are $\operatorname{Sp}(2, R)$ gauge invariant, in particular the $\mathrm{SO}(d, 2)$ is realized in the same unitary representation in all the derived $d$-dimensional theories. Likewise, it must be possible to compute various $\operatorname{Sp}(2, R)$ gauge invariant quantities and obtain the same or related results by using the different one-time field theories, including the interactions. Further computations along these lines, using the full power of interacting field theory, would help to strenghthen the case for two time physics, and perhaps help discover some of its utility by demonstrating that one could perform certain computations more easily by choosing a particular version of the field theory.

\section{Spin 1/2 field}

If we take $n=1$ in (20,21) then the physical state describes a spin $1 / 2$ field. The fermion $\psi^{M}$ is represented by a Dirac gamma matrix $\psi^{M}=\gamma^{M} / \sqrt{2}$, and position space now has an additional $\mathrm{SO}(d, 2)$ spinor index $<X, \alpha \mid$. The fermionic field is given by the probability amplitude $<X, \alpha \mid \Phi>=\Psi_{\alpha}(X)$. To satisfy the singlet OSp(1|2) conditions given in (20.21) we see that it is sufficient to impose the kinematic constraints

$$
\left(X \cdot \partial+\frac{d}{2}\right) \Psi_{\alpha}=0, \quad X^{2} \Psi_{\alpha}=0 .
$$

and the free field equation $(\gamma \cdot X \gamma \cdot \partial \Psi)_{\alpha}=0$. The second kinematic constraint follows from the property of the bra $<X, \alpha \mid X^{2}=0$. From

$$
(\gamma \cdot X \gamma \cdot \partial)^{2}=-X^{2} \partial^{2}+2(\gamma \cdot X)(\gamma \cdot \partial)\left(X \cdot \partial+\frac{d}{2}\right),
$$


we see $X^{2} \partial^{2} \Psi_{\alpha}=0$ need not be imposed as a separate free field equation. To include interactions consistently with the "kinematic" constraints we assume that the worldline version of the $\mathrm{OSp}(1 \mid 2)$ gauge theory is properly generalized by including background fields as in [10]. This permits the addition of source terms to the free field equation

$$
[\gamma \cdot X \gamma \cdot(\partial-i A) \Psi]_{\alpha}=h \Phi^{2 /(d-2)}(\gamma \cdot X \Psi)_{\alpha}+(\gamma \cdot X \xi)_{\alpha} .
$$

where $\xi_{\alpha}$ is any other fermion that does not blow up when $X^{2} \rightarrow 0$, and whose dimension is $\left(X \cdot \partial+\frac{d-2}{2}\right) \xi_{\alpha}=0$. The interacting field equation follows from varying the following Lagrangian

$$
L_{d+2}^{\Psi}=\bar{\Psi} \gamma \cdot X \gamma \cdot(\partial-i A) \Psi-h \Phi^{2 /(d-2)} \bar{\Psi} \gamma \cdot X \Psi+\bar{\Psi} \gamma \cdot X \xi
$$

The inclusion of the Yang-Mills gauge field $A_{M}(X)$ assumes that $\Psi$ is charged under the YangMills local internal symmetry. The scalar $\Phi$ must also have the correct charges to couple to the fermion with a non-zero coupling constant $h$, so the notation is schematic. We also assumed that the field $\Phi$ included on the right hand side may be of the type described in the previous section; if so this coupling would modify the field equations for $\Phi$ given in the previous section.

The form, and consistency of the interactions with the underlying $\operatorname{OSp}(1 \mid 2)$ gauge symmetry, are determined by applying $X \cdot \partial$ or $X \cdot \gamma$ on both sides of (84) and using the kinematic equations in (82) and (25). This also produces the conditions

$$
X^{2} A_{M}=0, \quad(X \cdot \partial+1) A_{M}=0, \quad X \cdot A=0
$$

on the gauge field. The same "kinematic" equations for the gauge field also follow from other independent considerations, including consistency of background fields with the $\operatorname{Sp}(2, R)$ gauge symmetry in the two-time worldline formalism [10], and the analysis in the following section for higher spinning fields.

Finally, it is important to note that (84) or (85) have a kappa type local fermionic symmetry given by

$$
\delta \Psi_{\alpha}=X_{M}\left(\gamma^{M} \kappa\right)_{\alpha}
$$

where $\kappa_{\alpha}(X)$ is any spinor in $d+2$ dimensions. To prove the kappa symmetry use $\gamma \cdot(\partial-i A)$ $\gamma \cdot X=-\gamma \cdot X \gamma \cdot(\partial-i A)+X \cdot(\partial-i A)+(d+2) / 2$ and apply the kinematic conditions (82). This means that only half of the fermions are physical, in accord with what is expected when the two-time theory is reduced from $d+2$ dimensions to $d$ dimensions.

In the case of free fields Dirac showed, with the parametrization given in (31), that the solution space of these equations is precisely the massless Dirac equation for a fermionic field in $d$ dimensions. This is also the conclusion reached in [4] by quantizing the $\operatorname{OSp}(1 \mid 2)$ worldline theory in the gauge $X^{+^{\prime}}(\tau)=1, P^{+^{\prime}}(\tau)=0,{\psi^{+}}^{\prime}(\tau)=0$. To show how the $d$ dimensional theory is embedded in $d+2$ we give here the field theory version of the gauge choice used in the 
worldline approach. We first fix the kappa symmetry so that it corresponds to the worldline fermionic gauge $\psi^{+^{\prime}}(\tau)=0$

$$
\left(\gamma^{+^{\prime}} \Psi\right)_{\alpha}=0 .
$$

Then use the parametrization (31) and the chain rule (32,33) to show that the operators applied on the gauge fixed $\Psi$ can be rewritten in the form

$$
\begin{aligned}
\gamma^{M} \partial_{M} \Psi & =\frac{1}{\kappa} \gamma^{\mu} D_{\mu} \Psi+\frac{1}{\kappa}\left(\gamma^{-^{\prime}}-x^{\mu} \gamma_{\mu}\right) \Psi \\
\gamma^{M} X_{M} \Psi & =-\kappa\left({\gamma^{-}}^{\prime}-x^{\mu} \gamma_{\mu}\right) \Psi
\end{aligned}
$$

and

$$
\begin{aligned}
& \gamma \cdot X \gamma \cdot(\partial-i A) \Psi=-\left(\gamma^{-^{\prime}}-x^{\mu} \gamma_{\mu}\right) \gamma^{\mu}\left(D_{\mu}-i A_{\mu}\right) \Psi \\
& +\left(\gamma^{-^{\prime}}-x^{\mu} \gamma_{\mu}\right)\left(2 \lambda-x^{2}\right)\left(\partial_{\lambda}-i A_{\lambda}\right) \Psi
\end{aligned}
$$

where $D_{\mu}=\partial_{\mu}+x_{\mu} \partial_{\lambda}$ as in (36). Inserting these forms in the interacting equation of motion we see that we remain with the overall factor $\left(\gamma^{-^{\prime}}-x^{\mu} \gamma_{\mu}\right)$ on both sides of the equation, but

since this is an ivertible matrix that satisfies $\left(\gamma^{-^{\prime}}-x^{\mu} \gamma_{\mu}\right)^{2}=x^{2}$, it can be removed from both sides. Furthermore by using $X^{2}=0$ we set $\lambda=x^{2} / 2$ which eliminates the last term in the last equation.

The result is the interacting massless Dirac field in $d$ dimensions, with $\mathrm{SO}(d, 2)$ conformal symmetry, in full agreement with the worldline theory approach. Therefore, the content of $(82,84)$ or $(85)$ using the $d$ dimensional coordinates (31) and kappa gauge (88) is the interacting massless fermionic field with $\mathrm{SO}(d, 2)$ conformal symmetry.

However, as discussed in 4 there are other gauge choices in two-time physics which would lead to other physical interpretations for the $\mathrm{SO}(d, 2)$ symmetry and of the dynamics from the point of view of a one-time observer. Using the corresponding parametrization for $X^{M}, \psi^{M}$ we fully expect that the two-time field equations (82) would yield the same richness of $d$ dimensional spin $1 / 2$ one-time physics, but now in the language of field theory.

\section{$6 \quad$ Vector and higher spin fields}

When $n=2,3, \cdots$ the fermions $\psi_{a}^{M}$ lead to higher spin particles. To display the spin components of the wavefunction we adapt the methods of [28] to the case of $\mathrm{SO}(d, 2)$. The $n$ anticommuting $\psi_{a}^{M}$ are represented in terms of $\mathrm{SO}(d+2)$ Dirac gamma matrices $\gamma_{\alpha \beta}^{M}$ acting in spinor space labelled by $\alpha=1, \cdots, 2^{(d+2) / 2}$. They are given in direct product form acting on the physical state with spin components $\left|\Phi_{\alpha_{1} \alpha_{2} \cdots \alpha_{a} \cdots \alpha_{n}}\right\rangle$

$$
\psi_{a}^{M}=\gamma^{*} \otimes \cdots \gamma^{*} \otimes \frac{1}{\sqrt{2}} \gamma^{M} \otimes 1 \otimes \cdots 1
$$


where the $\frac{1}{\sqrt{2}} \gamma^{M}$ is inserted in the $a^{\prime}$ th entry of the direct product, and $\gamma^{*}$ (analog of $\gamma_{5}$ in four dimensions) is the product of all $d+2$ gamma matrices $\gamma^{*}=i^{(d+2) / 2} \gamma^{0^{\prime}} \gamma^{1^{\prime}} \gamma^{0} \cdots \gamma^{d-1}$ such that $\left\{\gamma^{*}, \gamma^{M}\right\}=0$ and $\left(\gamma^{*}\right)^{2}=1$ (for simplicity, we assume even $d+2 \equiv 2 r$. If $d+2$ is odd the spinor space $\alpha$ is doubled to avoid $\gamma^{*}$ proportional to identity).

In this formalism the constraint $\psi_{[a} \cdot \psi_{b]}=0$ (for $n \neq 0$ ) on the physical state is solved by the following spin wavefunction. For even $n(n \neq 2)$ the spin wavefunction $\langle X| \Phi_{\alpha_{1} \alpha_{2} \cdots \alpha_{a} \cdots \alpha_{n}}>$ is a bosonic field written in terms of a $\mathrm{SO}(d, 2)$ tensor $F_{\text {indices }}(X)$ whose indices correspond to a Young tableau shaped like a rectangle, with $(d+2) / 2$ columns and $n / 2$ rows, as follows

$$
\begin{aligned}
\Phi_{\alpha_{1} \alpha_{2} \cdots \cdots \alpha_{n}}= & \left(\gamma^{M_{1}^{1} \cdots M_{(d+2) / 2}^{1}}\right)_{\alpha_{1} \alpha_{2}}\left(\gamma^{M_{1}^{2} \cdots M_{(d+2) / 2}^{2}}\right)_{\alpha_{3} \alpha_{4}} \cdots\left(\gamma^{M_{1}^{n / 2} \cdots M_{(d+2) / 2}^{n / 2}}\right)_{\alpha_{n-1} \alpha_{n}} \\
& \times F_{\left[M_{1}^{1} \cdots M_{(d+2) / 2}^{1}\right] ;\left[M_{1}^{2} \cdots M_{(d+2) / 2}^{2}\right] \cdots\left[M_{1}^{n / 2} \cdots M_{(d+2) / 2}^{n / 2}\right]} .
\end{aligned}
$$

The indices on $F_{\text {indices }}$ have permutation properties associated with $\mathrm{SO}(n)$ type Young tableaux : (i) the antisymmetric indices $\left[M_{1}^{i} \cdots M_{(d+2) / 2}^{i}\right]$ correspond to the column $i$, (ii) the $n / 2$ columns of indices for different $i$ 's are symmetrized with each other, (iii) under anti-symmetrization with one more index of a neighboring column the wavefunction vanishes

$$
F_{\left.\left[M_{1}^{1} \cdots M_{(d+2) / 2}^{1} ; M_{1}^{2}\right] \cdots M_{(d+2) / 2}^{2}\right] \cdots\left[M_{1}^{n / 2} \cdots M_{(d+2) / 2}^{n / 2}\right]}=0,
$$

(iv) to insure irreducibility under $\mathrm{SO}(d, 2)$ a vanishing trace for any pair of indices using $\eta^{M N}$ is required, symbolically $F_{\text {indices }} \cdot \eta=0$.

For odd $n$ the spin wavefunction is a fermionic field $\psi_{\text {indices }}^{\alpha}(X)$, whose indices correspond to the Young tableau described above with $(n-1) / 2$ columns, and there is one leftover spinor index $\alpha$, which satisfies the irreducibility condition

$$
\left(\gamma^{M_{1}^{1}}\right)_{\alpha \beta} \psi_{\left[M_{1}^{1} \cdots M_{(d+2) / 2}^{1}\right] ;\left[M_{1}^{2} \cdots M_{(d+2) / 2}^{2}\right] ; \cdots}^{\beta}=0 .
$$

For $n=2$, there is an exception since $q \neq 0$ is possible for an $\mathrm{SO}(2)$ singlet. Then it is possible to get a singlet (gauge invariant) of $\mathrm{OSp}(2 \mid 2)$ even though it is not necessarily neutral under the subgroup $\mathrm{SO}(2)$. This allows a more general solution for $\Phi_{\alpha_{1} \alpha_{2}}$ than the above. Imposing $\psi_{[1} \cdot \psi_{2]}|\Phi>=2 i q| \Phi>$ we find

$$
\begin{aligned}
\Phi_{\alpha_{1} \alpha_{2}}(X) & =\left(1+i \gamma^{*} \operatorname{sign}(q)(-1)^{p}\right)\left(\gamma^{M_{1} \cdots M_{p+2}}\right)_{\alpha_{1} \alpha_{2}} F_{M_{1} \cdots M_{p+2}}(X), \\
p & =\frac{1}{2}(d-2)-|q|=\text { integer. }
\end{aligned}
$$

Therefore, by adjusting the value of $q$ it is possible to obtain solutions that correspond to antisymmetric tensors $F_{M_{1} \cdots M_{p+2}}(X)$ with any of the values of $p$ in the set $\left\{-1,0,1, \cdots, \frac{1}{2}(d-2)\right\}$. If $q=0$ only the last value of $p$ is possible. This in contrast with the case of $n \geq 3$ for which only one solution is possible as given above.

For the rest of the discussion, for simplicity, we will specialize to the $n=2$ case, and furthermore concentrate on free fields so we will relax the conditions $X^{2} P^{2} \sim X \cdot \psi_{a} P \cdot \psi_{a} \sim 0$ 
of $(20,21)$ to $P^{2} \sim P \cdot \psi_{a} \sim 0$. The physical state condition $\psi_{a} \cdot P \sim 0$ requires that the $n=2$ wavefunction $F_{M_{1} \cdots M_{p+2}}(X)$ given in (97) is an on-shell field strength for a $p$-brane gauge potential $A_{M_{1} \cdots M_{p+1}}$

$$
F_{M_{1} \cdots M_{p+2}}(X)=\partial_{\left[M_{p+2}\right.} A_{\left.M_{1} \cdots M_{p+1}\right]}, \quad \partial^{M_{p+2}} \partial_{\left[M_{p+2}\right.} A_{\left.M_{1} \cdots M_{p+1}\right]}=0 .
$$

The additional physical state condition in $(20)$ requires a specific dimension

$$
X \cdot \partial F_{M_{1} \cdots M_{p+2}}=-\left(\frac{d-2}{2}+2-|q|\right) F_{M_{1} \cdots M_{p+2}}=-(p+2) F_{M_{1} \cdots M_{p+2}} .
$$

This equation holds provided the gauge field satisfies $(X \cdot \partial+p+1) A_{M_{1} \cdots M_{p+1}}=\partial_{\left[M_{1}\right.} U_{\left.M_{2} \cdots M_{p+1}\right]}$ for any $U_{M_{2} \cdots M_{p+1}}$. Through a gauge transformation $\delta A_{M_{1} \cdots M_{p+1}}=\partial_{\left[M_{1}\right.} \Lambda_{\left.M_{2} \cdots M_{p+1}\right]}$ one can eliminate $U$, hence $U$ is arbitrary. With the choice $U_{M_{2} \cdots M_{p+1}}=X^{M_{1}} A_{M_{1} \cdots M_{p+1}}$ the condition on $A_{M_{1} \cdots M_{p+1}}$ takes the gauge invariant form $X^{M_{p+2}} F_{M_{1} \cdots M_{p+2}}=0$.

The last equation in (99) may be modified to include interactions through a conserved $p$ brane current, so the combined equations (99,100) may be generalized to

$$
X^{M_{p+2}} F_{M_{1} \cdots M_{p+2}}=0, \quad \partial^{M_{p+2}} F_{M_{1} \cdots M_{p+2}}=J_{M_{1} \cdots M_{p+1}}
$$

The last equation contracted with either $X^{M_{1}}$ or $\partial^{M_{1}}$ shows that the brane current must be conserved, satisfy an additional constraint, and be of definite dimension

$$
\partial^{M_{1}} J_{M_{1} \cdots M_{p+1}}=0, \quad X^{M_{1}} J_{M_{1} \cdots M_{p+1}}=0, \quad(X \cdot \partial+p+1) J_{M_{1} \cdots M_{p+1}}=0 .
$$

The first equation in (101) is "kinematics" and the last is dynamics. The dynamical equation follows from a varying the Lagrangian

$$
L_{d+2}^{A}=-\frac{1}{4} F_{M_{1} \cdots M_{p+2}} F^{M_{1} \cdots M_{p+2}}+A^{M_{1} \cdots M_{p+1}} J_{M_{1} \cdots M_{p+1}}
$$

which has the gauge invariance for a $p+1$ gauge potential.

In the case of a vector potential we may identify it with the Yang-Mills gauge potential that appeared in the previous section and which coupled to the charged scalars or fermions. Then the current $J_{M}$ need not be included as an additional source at it follows from the gauge couplings in $L_{d+2}^{\Phi}$ or $L_{d+2}^{\Psi}$.

The $p$ brane potential $A_{M_{1} \cdots M_{p+1}}$ satisfies similar constraints to those of $J_{M_{1} \cdots M_{p+1}}$ after fixing some gauge symmetries. Consider fixing the gauge $X^{M_{1}} A_{M_{1} \cdots M_{p+1}}=0$. Then the first equation in (101) reduces to $(X \cdot \partial+p+1) A_{M_{1} \cdots M_{p+1}}=0$, which requires $A_{M_{1} \cdots M_{p+1}}$ to have a definite dimension. Despite the gauge choice there still remains gauge symmetry under $\delta A_{M_{1} \cdots M_{p+1}}=$ $\partial_{\left[M_{1}\right.} \Lambda_{\left.M_{2} \cdots M_{p+1}\right]}$, for all $\Lambda_{M_{2} \cdots M_{p+1}}$ that have dimension $p$, i.e. $(X \cdot \partial+p) \Lambda_{M_{2} \cdots M_{p+1}}=0$. This is sufficient gauge symmetry to further fix the gauge of $A_{M_{1} \cdots M_{p+1}}$ since it now has a definite dimension. Thus, through these gauge choices we may take a $A_{M_{1} \cdots M_{p+1}}$ that satisfies constraints similar to those of the current

$$
X^{M_{1}} A_{M_{1} \cdots M_{p+1}}=0, \quad \partial^{M_{1}} A_{M_{1} \cdots M_{p+1}}=0, \quad(X \cdot \partial+p+1) A_{M_{1} \cdots M_{p+1}}=0,
$$


while the dynamics simplifies to the gauge fixed form

$$
\partial^{M} \partial_{M} A_{M_{1} \cdots M_{p+1}}=J_{M_{1} \cdots M_{p+1}} .
$$

If we specialize to $n=2$ and $p=0$ (or $|q|=(d-2) / 2$ ) the physical state is a vector gauge field $A_{M}$ that satisfies the gauge invariant equations for $F_{M N}=\partial_{M} A_{N}-\partial_{N} A_{M}$

$$
\begin{aligned}
X^{M} F_{M N}=0, & \partial^{M} F_{M N}=J_{N}, \\
X^{M} J_{M}=0, & \partial^{M} J_{M}=0, \\
(X \cdot \partial+2) F_{M N}=0, & (X \cdot \partial+1) J_{M}=0 .
\end{aligned}
$$

For the fixed gauge described above these equations become

$$
\begin{aligned}
& (X \cdot \partial+1) A_{M}=0, \quad X^{M} A_{M}=0, \quad \partial^{M} A_{M}=0, \quad \partial^{M} \partial_{M} A_{N}=J_{N}, \\
& (X \cdot \partial+1) J_{M}=0, \quad X^{M} J_{M}=0, \quad \partial^{M} J_{M}=0 .
\end{aligned}
$$

These coincide with equations that appear in Dirac's paper [1] for the vector gauge potential. They also are in agreement with the results of the background field approach introduced in [10.

Following Dirac, if we take $d+2=6$ dimensions, the solution of these field equations in the parametrization of eq.(31) is precisely equivalent to Maxwell's equations for a gauge potential $A_{\mu}(x)$ in $d=4$ dimensions identified as part of the six dimensional $A_{M}(X)$. The conformal symmetry of Maxwell's theory in four dimensions is none other than the $\mathrm{SO}(4,2)$ Lorentz symmetry in six dimensions.

As we have emphasized in the previous sections the parametrization of eq.(31) is connected to one of the possible gauge choices in two-time physics. Parametrizations that are related to other gauge choices would reveal other physical content in the $d$ dimensional field theory.

\section{Gravity}

All of the interacting Lagrangians above can be coupled to gravity. To do so we follow the prescription obtained in [10]. In the usual way we need a metric $G_{M N}(X)$ or vielbein $E_{M}^{a}(X)$ and a spin connection for $\mathrm{SO}(d, 2) \omega_{M}^{a b}(X)$ in $d+2$ dimensions, but we also need an additional vector $V^{M}(X)$ constructed from a potential $W(X)$. These fields satisfy the following kinematic equations

$$
£_{V} G^{M N}=-2 G^{M N}, \quad V^{M}=\frac{1}{2} G^{M N} \partial_{N} W, \quad G^{M N} \partial_{M} W \partial_{N} W=4 W
$$

where $£_{V} G^{M N}$ is the Lie derivative $£_{V} G^{M N}=V \cdot \partial G^{M N}-\partial_{K} V^{M} G^{K N}-\partial_{K} V^{N} G^{K M}$. Furthermore the kinematic conditions we had earlier for the various fields now take the form

$$
\begin{aligned}
£_{V} \Phi & =-\frac{d-2}{2} \Phi, \quad £_{V} \Psi_{\alpha}=-\frac{d}{2} \Psi_{\alpha}, \quad V^{M} F_{M N}=0, \\
W(X) \Phi & =0, \quad W(X) \Phi=0, \quad W(X) A_{M}=0 .
\end{aligned}
$$


where the ordinary derivatives in the Lie derivative $£_{V}$ should be replaced by covariant derivatives consistent with a local Lorentz symmetry $\mathrm{SO}(d, 2)$ in tangent space. Thus, wherever there was an explicit $X^{M}$ in flat space, it is now replaced by $V^{M}(X)$ and wherever there was a YangMills derivative $\partial_{M}+A_{M}$ it is now promoted to a $\mathrm{SO}(d, 2)$ covariant derivative $\partial_{M}+\omega_{M}+A_{M}$. Using these modifications the Lagrangians $L_{d+2}^{\Phi}, L_{d+2}^{\Psi}, L_{d+2}^{A}$ constructed earlier in this paper are generalized to couple to gravity consistently with the underlying $\operatorname{OSp}(n \mid 2)$ gauge symmetries of two-time physics. They should also be multiplied by a volume factor $\sqrt{G}=\operatorname{det} E$ that satisfies $£_{V} \sqrt{G}=(d+2) \sqrt{G}$ as it follows from (111).

Next we would like to write down a Lagrangian $L_{d+2}^{G}$ for the gravitational sector. But first we will deal with the kinematic constraints in (111) by rewriting them in tangent space using the vielbein and spin connection and giving them a more geometrical meaning. In particular since the spin connection is a gauge field its field strength (the curvature) must satisfy

$$
V^{M} R_{M N}^{a b}=0, \quad R_{M N}^{a b}=\partial_{M} \omega_{N}^{a b}-\partial_{N} \omega_{M}^{a b}+\left[\omega_{M}, \omega_{N}\right]^{a b} .
$$

like other gauge fields in (112). Similarly, the vielbein can also be viewed as a gauge field. We define the covariant derivative of the vielbein with respect to the spin connection

$$
D_{M} E_{N}^{a}=\partial_{M} E_{N}^{a}+\omega_{M b}^{a} E_{N}^{b}
$$

The torsion tensor is given by

$$
T_{M N}^{a}=D_{M} E_{N}^{a}-D_{N} E_{M}^{a}
$$

So, we also take the torsion tensor to satisfy the transversality condition, as a kinematic condition

$$
V^{M} T_{M N}^{a}=0
$$

We define the Lie derivative of the vielbein by including the covariant derivative using the spin connection; it may be rewritten in terms of the torsion as follows

$$
\begin{aligned}
£_{V} E_{M}^{a} & =V^{N} D_{N} E_{M}^{a}+\partial_{M} V^{N} E_{N}^{a} \\
& =V^{N} D_{[N} E_{M]}^{a}+V^{N} D_{M} E_{N}^{a}+\partial_{M} V^{N} E_{N}^{a} \\
& =V^{N} T_{N M}^{a}+D_{M} V^{a} \\
& =D_{M} V^{a}
\end{aligned}
$$

we have used the transversality condition on the torsion and defined $V^{a}=E_{M}^{a} V^{M}$, and $D_{M} V^{a}=$ $\partial_{M} V^{a}+\omega_{M}^{a b} V_{b}$. If $£_{V} E_{M}^{a}$ is contracted with another vielbein we obtain $£_{V} G_{M N}$ in the form

$$
£_{V} G_{M N}=2 £_{V} E_{M}^{a} E_{N a}=2 D_{M} V^{a} E_{N a} .
$$

Due to the condition (111) this quantity is $2 G_{M N}$. Multiplying both sides by $E^{N a}$ we find

$$
£_{V} E_{M}^{a}=E_{M}^{a},
$$


with

$$
E_{N}^{a}=D_{M} V^{a}=\partial_{M} V^{a}+\omega_{M}^{a b} V_{b}
$$

This form has been previously suggested in [13]), we derived it here from the homothety conditions (111) obtained in the worldline formalism [10].

Thus, the vielbein constructed in this way satisfies the kinematic condition automatically while it is fully determined by the arbitrary functions $V^{a}(X)$ and $\omega_{M}^{a b}(X)$. The only condition on the functions $V^{a}, \omega_{M}^{a b}$ is that the curvature and torsion be transverse to $V^{M}$. Modulo this condition the vielbein, and metric $G_{M N}$ are determined. This form solves automatically the first equation in (111). Similarly, the last equation in (111) can be rewritten in terms of $V^{a}$

$$
W=V^{a} V_{a}
$$

There remains the second equation in (111) that now takes the form

$$
V_{a}=\frac{1}{2} E_{a}^{M} D_{M}\left(V_{b} V^{b}\right)=E_{a}^{M}\left(D_{M} V^{b}\right) V_{b}=E_{a}^{M} E_{M}^{b} V_{b}
$$

which is an identity since $E_{a}^{M} E_{M}^{b}=\delta_{a}^{b}$. Thus, all kinematic conditions for the gravitational field are fully solved by the arbitrary functions $V^{a}(X)$ and $\omega_{M}^{a b}(X)$, and the definition of metric through $E_{M}^{a}=D_{M} V^{a}$. Note also that we can rewrite the torsion as follows

$$
T_{M N}^{a}=D_{[M} D_{N]} V^{a}=R_{M N}^{a b} V_{b}, \quad \text { or } \quad T_{c d}^{a}=R_{c d}^{a b} V_{b}
$$

Therefore, the torsion is obtained from the curvature (the spin connection included torsion).

From this construction we may deduce (using $£_{V} E_{M}^{a}=E_{M}^{a}$ and $£_{V} R_{M N}^{a b}=-2 R_{M N}^{a b}$ as any other gauge field strength)

$$
\begin{aligned}
£_{V} V^{a} & =V^{M} D_{M} V^{a}=V^{M} E_{M}^{a}=V^{a}, \\
£_{V} R_{c d}^{a b} & =£_{V}\left(E_{c}^{M} E_{d}^{N} R_{M N}^{a b}\right)=-4 R_{c d}^{a b}, \\
£_{V} T_{c d}^{a} & =-3 T_{c d}^{a} .
\end{aligned}
$$

The transversality conditions $V^{M} R_{M N}^{a b}=0$ on the curvature and torsion may be rewritten in tangent space

$$
V^{c} R_{c d}^{a b}=0
$$

while $V^{c} T_{c d}^{a}=0$ is automatically satisfied thanks to (127). This is the only remaining kinematic condition on the gravitational background as long as the primary building blocks are $V^{a}$ and $\omega_{M}^{a b}$.

We now turn our attention to the Lagrangian in the gravitational sector that generates the dynamics for gravity (i.e. impose the analog of the Einstein equations) in two-time physics. Naively the Lagrangian would be given by the Riemann curvature scalar $R=R_{a b}^{a b}$ but we must 
seek a modification in light of the constraints generated by $£_{V}$ as in (111, 131, 112). Consistent coupling with these constraints requires the form

$$
L_{d+2}^{G} \sim(\operatorname{det} E) R_{a b}^{a b} \Phi^{2(d-4) / d-2}
$$

where $\Phi$ is one (or a combination) of the scalar fields described earlier. Typically the scalar that appears in the overall factors in the Lagrangians we constructed up to now would be identified as the dilaton.

\section{Discussion}

Combining the Lagrangians for scalars, spinors, vectors and gravitons we have a total Lagrangian that generates the dynamical equations of motion through a variational principle, and couples all the fields to one another

$$
\begin{aligned}
L_{d+2} & =L_{d+2}^{G}+L_{d+2}^{A}+L_{d+2}^{\psi}+L_{d+2}^{\phi} \\
L_{d+2}^{G} \sim(\operatorname{det} E) \Phi^{2(d-4) / d-2} R_{a b}^{a b} & \\
L_{d+2}^{A} & =-\frac{1}{4}(\operatorname{det} E) \Phi^{2(d-4) / d-2} F_{M N} F_{K L} G^{M K} G^{N L} \\
L_{d+2}^{\Psi} & =(\operatorname{det} E)\left[\bar{\Psi} \gamma \cdot V \gamma \cdot(\partial-i A) \Psi-h \Phi^{2 /(d-2)} \bar{\Psi} \gamma \cdot V \Psi+\bar{\Psi} \gamma \cdot V \xi\right] \\
L_{d+2}^{\Phi} & =-\frac{1}{2} \Phi \partial_{M}\left(\sqrt{G} G^{M N} \partial_{N} \Phi\right)-\lambda \frac{(d-2)}{2 d} \Phi^{2 d /(d-2)} \sqrt{G} .
\end{aligned}
$$

The dynamical equations thus obtained must be supplemented with the kinematic conditions in (112) and (131). In the gravitational sector $V^{a}, \omega_{M}^{a b}$ are the primary fields, not $E_{M}^{a}$ or $G_{M N}$.

As discussed above Dirac's program for coming down from $d+2$ dimensions to $d$ dimensions can be implemented through many possible paths, ending up with a choice of some $d$ dimensions embedded in $d+2$ dimensions. In this way one arrives at different looking but non-trivially related interacting field theories in $d$ dimensions. This is the new lesson learned from two-time physics.

As mentioned earlier one may consider several scalars, spinors, vectors etc., and build a $d+2$ Lagrangian that would reproduce the Standard Model in one of the $d=4$ versions of a $d+2=6$ dimensional theory in two-time physics. The natural choice of 4 dimensions among the 6 is the one given in (31) since that is the one that corresponds to the massless relativistic particle. It would be interesting to find out what one can learn from the other choices of 4 dimensions that would produce dual versions of the Standard Model. In particular, can one discover non-perturbative phenomena, relations among parameters, or new measurable effects of the standard theory in particle physics? These questions remain to be investigated in general as well as for the Standard Model itself.

The two-time formulation presented here and in [2]-[10] has properties that touches upon other popular but little understood concepts in the current literature. Among them duality, 
holography, AdS-CFT, background independence are ideas that can be seen to be present in two-time physics is some generalized sense. Holography can be compared to the fact that $d$ dimensions, which can be thought of as a surface around the bulk of $d+2$ dimensions, is sufficient to describe all of the physics contained in the bulk. In our version of holography we go down two dimensions rather than one and therefore there is not just one $d$ dimensional "surface" but many, and this connects to our version of "duality". Duality can be compared to the many versions of $d$ dimensional theories that are related and actually represent the same $d+2$ dimensional theory (an analogy to M-theory). We have already given a concrete example of the usual AdS-CFT correspondence at the end of section (4.2), as seen from the point of view of two-time physics, and this could be generalized to more interesting cases. Finally concepts of background independence are present since one could start with a $d+2$ theory without backgrounds and end up with a theory in $d$ dimensions with many possible curved backgrounds.

We need to end on a down note, but hopefully a stimulating one. The formulation of two-time physics in field theory presented here is incomplete. The fact that the subsidiary "kinematic" conditions are not derived directly as an equation of motion from the field theory Lagrangian is a sign that the formulation is incomplete. Surely one could introduce Lagrange multipliers to impose these conditions, but this seems artificial. Introducing a delta function $\delta\left(X^{2}\right)$ or $\delta\left(V^{2}\right)$ in the action built from the Lagrangian above is also just as artificial, and it still misses the other kinematic constraints due to $£_{V}$. Rather, a gauge principle that implements the underlying $\operatorname{Sp}(2, R)$ or $\operatorname{OSp}(n \mid 2)$ gauge symmetry directly in field theory is the needed ingredient. This would generate all the kinematic or dynamic constraints simultaneously, as it does in the worldline formalism. In this sense the worldline formalism seems more fundamental at the current stage.

Once the field theory formulation is completed it would then be possible to investigate with more confidence second quantization in the formalism of two-time physics, and try to establish the validity of the duality relations among the d-dimensional theories at the second quantized level. Some such duality is expected, but the correct ordering of operators (or corresponding anomalies) may need further understanding.

To implement the $\operatorname{Sp}(2, R)$ gauge symmetry in field theory suggested above it may be more natural to consider fields that are functions over phase space $\Phi(X, P)$. This appears to go in the direction of non-commutative geometry, but with specific goals that are not currently part of the thinking in non-commutative geometry. Perhaps it would be helpful to investigate in this direction to complete the field theoretic formulation of two-time physics.

\section{References}

[1] P.A.M Dirac, Ann. Math. 37 (1936) 429. 
[2] I. Bars, C. Deliduman and O. Andreev, Phys. Rev. D58 (1998) 066004, or hep-th/9803188; I. Bars, Phys. Rev. D58 (1998) 066006, or hep-th/9804028; S. Vongehr, "Examples of black holes in two time physics", hep-th/9907077; for a review see, I. Bars, hep-th/9809034.

[3] I. Bars, Phys. Rev. D59 (1999) 045019, or hep-th/9810025.

[4] I. Bars and C. Deliduman, Phys. Rev. D58 (1998) 106004, or hep-th/9806085.

[5] I. Bars, C. Deliduman and D. Minic, Phys. Rev. D59 (1999) 125004 or hep-th/9812161.

[6] I. Bars, C. Deliduman and D. Minic, Phys. Lett. B457 (1999) 275 or hep-th/9904063; I. Bars, "A toy-M-model", in preparation.

[7] I. Bars, C. Deliduman and D. Minic, Phys. Lett. B466 (1999) 135 or hep-th/9906223.

[8] L. Andrianopoli, M. Derix, G. W. Gibbons, C. Herdeiro, A. Santambrogio, A. Van Proeyen, "Isometric embeddings of BPS branes in flat spaces with two times", hep-th/9912049.

[9] I. Bars, "A toy-M-model", in preparation.

[10] I. Bars, "Two-time physics with gravitational and gauge field backgrounds", hepth/0002140.

[11] H. A. Kastrup, Phys. Rev. 150 (1966) 1183.

[12] G. Mack and A. Salam, Ann. Phys. 53 (1969) 174.

[13] C. R. Preitschopf and M. A. Vasilev, hep-th/9812113.

[14] M. Duff and M. P. Blencowe, Nucl. Phys. B310 (1988)387.

[15] I. Bars, "Duality and hidden dimensions", in the proceedings of the conference Frontiers in Quantum Field Theory, Toyonaka, Japan, Dec. 1995, Ed. (Itoyama et. al.), page. 52, hep-th/9604200; I. Bars Phys. Rev. D54 (1996) 5203.

[16] C. Vafa, Nucl. Phys. B469 (1996) 403.

[17] I. Bars, Phys. Rev. D55 (1997) 2373 or hep-th/9607112; hep-th/9608061; Phys. Lett. B403 (1997) 257 or hep-th/9704054. I. Bars, Phys. Rev. D56 (1997) 7954 or hep-th/9706185.

[18] D. Kutasov and E. Martinec, Nucl. Phys. B477 (1996) 652; Nucl. Phys. B477 (1996) 675.

[19] H. Nishino and E. Sezgin, Phys. Lett. B388 (1996) 569.

[20] I. Bars and C. Kounnas, Phys. Lett. B402 (1997) 25; Phys. Rev. D56 (1997)3664.

[21] E. Sezgin, Phys. Lett. B403 (1997) 265. 
[22] I. Bars and C. Deliduman, Phys. Rev. D56 (1997) 6579 or hep-th/9707215; Phys. Lett. B417 (1998)24 or hep-th/9710066.

[23] E. Sezgin and Rudychev, hep-th/9711128; Phys. Lett. B424 (1998) 60.

[24] H. Nishino, Phys. Lett. B426 (1998) 64 or hep-th/9710141; Nucl. Phys. B542 (1999) 217 or hep-th/9807199; hep-th/9901104.

[25] J. Maldacena, Adv. Theor. Math. Phys. 2 (1998) 231 or hep-th/9711200.

[26] S. S. Gubser, I. R. Klebanov and A. M. Polyakov, Phys. Lett. B428 (1998) 105 or hepth/9802109.

[27] E. Witten, Adv. Theor. Math. Phys. 2 (1998) 253 or hep-th/9802150.

[28] P. Howe, S. Penati, M. Pernici, P.K. Townsend, Phys. Lett. B215 (1988) 555; Class. Quant. Grav. 6 (1989) 1125. 